\title{
Macroscopic and Microscopic Structure of Electromagnetic Wakefield
}

\author{
H. Lin ${ }^{*}$, C.P. Liu, B.F. Shen and Z.Z. Xu \\ State Key Laboratory of High Field Laser Physics, Shanghai Institute of Optics and Fine Mechanics, P.O. Box 800-211, \\ Shanghai 201800, China
}

\begin{abstract}
Applying various stimuli to excite large-amplitude electrostatic structure within plasmas is a basic idea of plasma-based acceleration. However, because these stimuli are usually magnetized, whether or not their wakes within plasmas are purely electrostatic should be cautiously treated. By strict theory on self-consistent fields of charged particles, we make detailed investigations on the wakes of those magnetized stimuli and the acceleration by electromagnetic wakes.
\end{abstract}

Keyowrds: Plasma wakefield.

\section{INTRODUCTION}

The generation of high-energy charged particles from plasmas has been an appealing issue of plasma physics for decades. In 1970s, authors found from their computer simulation on two-stream instability [1-5], that electron phase-space distribution function display a hole structure when self-consistent field is set up within plasmas. Such a hole structure reflects the population of some lower energy electrons being suppressed while that of some higher energy electrons being elevated, and hence is a signal of the generation of high-energy charged particles, or of particle acceleration. It has also been described by some authors with "negative temperature" conception [6]. Some authors have noticed that a temperature profile, which is time-space varying, is more appropriate than a constant temperature to describe plasmas [7]. All of these earlier works have clearly indicated that plasma is an effective matrix for generating high-energy charged particles.

On the other hand, at the end of 1970s, Tajima and Dawson definitely proposed a notion which was plasmabased particle acceleration [8]. This notion stresses that plasma density wave can play a role of traditional accelerator. Because the plasma density wave is closely related to self-consistent electrostatic field within plasmas, this stimulates a lot of investigations on how to set up largeamplitude electrostatic wave within plasmas via various stimuli [9-31]. Two familiar conceptions, laser wakefield [13] and plasma wakefield [12,14], are typical examples of such a large-amplitude electrostatic wave. In 1980s, authors have set up basic 1-D theories on these two conceptions [12-14]. Then, during following decades, a lot of investigations have been addressed to various wakefieldrelated problems [15-31].

Despite so many related investigations on so-called wakefield, there still exists a basic question. Does a realistic

*Address correspondence to this author at the State Key Laboratory of High Field Laser Physics, Shanghai Institute of Optics and Fine Mechanics, P.O. Box 800-211, Shanghai 201800, China; Tel: 86-21-69918266;

Fax: 86-21-69918021; E-mail: linhai@ siom.ac.cn
3-D plasma electrostatic wave exist? Some authors have found from particle-in-cell (PIC) simulation, that the driven plasma density wave is accompanied by a similar magnetic energy density wave [32]. Because earlier 1-D theories [1214] cannot include magnetic field [12-14], this implies that we should set up a stricter theory on wakefields of various stimuli rather than simply treat them as electrostatic structures. A notable fact is that the stimuli to excite these wakefields usually do not correspond to zero self-consistent magnetic field. For example, laser pulse, (the stimulus driving the laser wakefield,) has a laser magnetic field and hence is a "magnetized" stimulus. Being stored usually in magnetic apparatus such as storage-ring, an electron beam is also a magnetized stimulus to excite the plasma wakefield. These "magnetized" stimuli force us to carefully treat their wakefields. Some authors have noticed that these wakefields are electromagnetic and set up a related nonlinear theory based on fluid approximation [27,39]. Moreover, some efforts have been paid to experimentally probe the magnetic fields structure of wakefields [33]. But the stress of their approximated fluid theory [27] is not focused on magnetic structure of every wake and hence does not predict the latter results found from the PIC simulation [32].

Earlier investigations displaying phase space holes [1-6] have revealed that electromagnetic self-consistent field can also lead to high-energy charged particles. High-energy particles generated from magnetic reconnection [32,34-36] also suggest that the particle acceleration should not merely be related to the electrostatic wakefield but is also available for electromagnetic wakefield. The particle acceleration, or the generation of high-energy particles, from the electromagnetic wakefield is a part of the purpose of this work. Strictly speaking, for a realistic "magnetized" stimulus, if its wake is "automatically" taken as an electrostatic one, the strength of such an electrostatic wake might be greatly overestimated and hence the estimation on some aspects of acceleration quality might be very optimistic.

The purpose of this work is to present a stricter basic method, which is universally applicable to plasma physics, and then put the investigations on the magnetic wakefield on 
a firm basis. The work is arranged as follows: By strictly analyzing several basic methods, we display a firm basic method in section.II. Subsection.III.A is for the applications of this firm basis to 3-D aperiodic electrostatic structure. The applications of this firm basis to the electromagnetic wakefield is conducted in subsection.III.C. Section.IV is a brief summary.

\section{BASIC METHODS}

Many textbooks [43-45] have displayed clearly a fundamental fact: Liouville theorem and Hamilton's equations

$$
\begin{aligned}
& d_{t} f(r(t), p(t), t)=0 \\
& d_{t} r(t)=\frac{\partial H}{\partial p(t)}=v(t) ; d_{t} p(t)=-\frac{\partial H}{\partial p(t)}
\end{aligned}
$$

will lead to well-known Vlasov equation (VE). Maybe someone will find that according to Klimontovich-Dupree method [45], a functional

$$
N(x, v, t) \equiv \sum_{i} \delta\left(x-x_{i}(t)\right) \delta\left(v-d_{t} x_{i}(t)\right)
$$

in which $x$ and $v$ are independent of $t$, meets $\mathrm{VE}$ and hence conclude that the $\mathrm{VE}$ is defined over $(x, v, t)$-space. However, their method can also be extended to the following functional

$$
N\left(x(t), d_{t} x(t), t\right) \equiv \sum_{i} \delta\left(x(t)-x_{i}(t)\right) \delta\left(d_{t} x(t)-d_{t} x_{i}(t)\right) .
$$

One can find that it also meets a VE defined over $(x(t), v(t), t)$-space. Therefore, for generality, we take $\mathrm{VE}$ as being defined over $(x(t), v(t), t)$-space.

This fundamental fact reminds us that VE is for an element whose trajectory in phase space is $[r(t), p(t)]$. Strict expression of VE should outstand time-dependence of $r(t)$ and $v(t)$

$\partial_{t} f(t, r(t), v(t))+d_{t} r(t) * \partial_{r(t)} f(t, r(t), v(t))$

$+d_{t} v(t) * \partial_{v(t)} f(t, r(t), v(t))=0$

In terms of fluid mechanics, VE and its fluid derivations are expressed by Lagrangian variables. On the other hand, Maxwell equations (MEs) are often expressed by Eulerian variables $(R, t)=(x, y, z, t)$, where $R$ and $t$ are independent variables.

Obviously, according to standard definition of fluid velocity (see standard textbooks [45] )

$u(x, t)=\frac{\int \sum_{i} v \delta\left(x-x_{i}(t)\right) \delta\left(v-d_{t} x_{i}(t)\right) d^{3} v}{\int \sum_{i} \delta\left(x-x_{i}(t)\right) \delta\left(v-d_{t} x_{i}(t)\right) d^{3} v}$,

we can find that there is usually $\Delta(x, v, t)=v-u(x, t) \neq 0$. If we try to find the strict solution of a VE from a power series of $\Delta$, we can formally write a trial solution $\ln f=\sum_{i \geq 0}\left[c_{2 i}(x, t) *(\Delta \cdot \Delta)^{i}+c_{2 i+1}(x, t) *(\Delta \cdot \Delta)^{i} *\left(\overleftarrow{e_{\Delta}} \cdot \Delta\right)\right],(7)$

where $\overleftarrow{e_{\Delta}}$ is unit vector along $\Delta$. Also, this power series can be transformed in terms of $p(v)-p(u(x, t))$, where $p(v a r)=\frac{v a r}{\sqrt{1-(\text { var })^{2}}}$. Inserting it back into a VE and comparing the coefficients of terms $\Delta^{j}$, we can easily find that for all terms $126 \Delta^{j}$ terms, there is

$$
\begin{aligned}
& \left\{\partial_{t} c_{j}(x, t)+d_{t} x * \nabla_{x} c_{j}(x, t)\right\} \\
& +(j+1) * c_{j+1}(x, t) *\left\{\left[\partial_{t} p(u(x, t))\right.\right. \\
& \left.\left.+d_{t} x * \nabla_{x} p(u(x, t))\right]-[E(x, t)+u(x, t) \times B(x, t)]\right\} \\
& =0.8
\end{aligned}
$$

Here, we have used the fact $\Delta \times B \cdot \Delta=0$. Obviously, a set of functions $c_{i}$ and $u$, if they meet

$$
\begin{aligned}
& \left.\partial_{t} p(u(x, t))+d_{t} x * \nabla_{x} p(u(x, t))\right] \\
& -[E(x, t)+u(x, t) \times B(x, t)]=0 ; \\
& \partial_{t} c_{j}(x, t)+d_{t} x * \nabla_{x} c_{j}(x, t)=0,
\end{aligned}
$$

they can yield a strict solution of the VE according to Eq. (7). Note that Eq. (9) is expressed by Lagrangian variables $(r(t), t)$. Clearly, if expressing this equation in terms of Euler variables, there will be

$\left.\partial_{t} p(u(X, t))\right]+[E(X, t)+u(X, t) \times B(X, t)]=0$.

Moreover, Eq. (10) is indeed $d_{t} c_{j}=0$ and hence means $c_{j}(x, t) \equiv c_{j}(x(0), 0)$.

Above treatment differs from conventional fluid treatment, in which equations of different orders of momentums $p$ are derived (by timing $p^{i}(v)$ with the VE and then integrating them over $v$-space) and form a closed equation set whose member is of infinite number. According to the conventional fluid treatment, an equation of fluid momentum,

$$
\begin{aligned}
& \partial_{t} p(x(t), t)+u(x(t), t) * \nabla_{x(t)} p(x(t), t) \\
& +[E(x(t), t)+u(x(t), t) \times B(x(t), t)], \\
& +\frac{1}{n} \nabla \cdot \text { Pressure }=0
\end{aligned}
$$

can be derived. Here,

Pressure $=\int(v-u) *\left(\frac{v}{\sqrt{1-v^{2}}}-p\right) f d^{3} v$. Note that the fluid momentum $p(x(t), t)$ differs from $\frac{u}{\sqrt{1-u^{2}}}$ in finite temperature case. In the conventional fluid treatment, people will seek for the equation of Pressure or that of the momentum of $p$ in next order. Actually, the conventional 
fluid treatment describe the charged particles with their momemtums of $p$ in different orders. In contrast, in above treatment, these $c_{i}$, and $u$, describe the system to be more straightforward. More important, two treatments are of a fundamental discrepancy. In the conventional fluid treatment, every order of momentum depends on those of higher order. This will inevitably invoke truncation approximation when seeking the exact momentum at specified order. In contrast, above treatment is free from the truncation approximation.

In the finite temperature case, the fluid momentum $p(x(t), t)$ differs from $\frac{u}{\sqrt{1-u^{2}}}$. In addition, according to the definition Eq. (6), $u(x(t), t)$ also differs from $d_{t} x$ when the temperature is non-zero. Both $p(x(t), t)-\frac{u}{\sqrt{1-u^{2}}}$ and $u(x(t), t)-d_{t} x$ are functions of the temperature. Pr essure is also a function of the temperature. In order to derive an equation of $u$ from the equation

$$
\begin{aligned}
& \partial_{t} p(x(t), t)+u(x(t), t) * \nabla_{x(t)} p(x(t), t) \\
& +[E(x(t), t)+u(x(t), t) \times B(x(t), t)], \\
& +\frac{1}{n} \nabla \cdot \text { Pr essure }=0
\end{aligned}
$$

we should make equal approximation on those temperaturedependent terms. Namely, if Pressure is taken as negligible because it is temperature-dependent, then we should also take $p(x(t), t)-\frac{u}{\sqrt{1-u^{2}}}$ and $u(x(t), t)-d_{t} x$ as negligible. Guided by this equal approximation spirit, we can derive Eq. (9) from the equation

$$
\begin{aligned}
& \partial_{t} p(x(t), t)+u(x(t), t) * \nabla_{x(t)} p(x(t), t) \\
& +[E(x(t), t)+u(x(t), t) \times B(x(t), t)] . \\
& +\frac{1}{n} \nabla \cdot \operatorname{Pr} \text { essure }=0
\end{aligned}
$$

The convective operator $u(x(t), t) * \nabla_{x(t)}$ is also temperature dependent. When neglecting Pressure, for same reason, some part of $u(x(t), t) * \nabla_{x(t)}$ should be neglected. Actually, even for the fluid velocity expressed by Euler variable, $u(R, t)=\frac{\sum_{i} d_{t} r_{i} \delta\left(R-r_{i}(t)\right)}{\sum_{i} d_{t} r_{i} \delta\left(R-r_{i}(t)\right)}$, if directly applying $\partial_{t}$ on it we can find when $T=0$, any $d_{t} r_{i}$ (if $R-r_{i}(t)$ is satisfied) can be shifted out of the summation sign $\sum$ (because it is equal to a quantity independent of the subindex $i$, or $u(R, t))$. This leads to that when $T=0$, the equation satisfied by $u(R, t)$ is

$$
\partial_{t} p(u(R, t))=E(R, t)+u(R, t) \times B(x(t), t)
$$

rather than

$$
\begin{aligned}
& \partial_{t} p(u(R, t))+u(R, t) \cdot \nabla_{R} p(u(R, t)) \\
& =E(R, t)+u(R, t) \times B(x(t), t)
\end{aligned} .
$$

Obviously, if using conventional fluid treatment, we will first obtain the equation of the fluid momentum $p(x(t), t)$ and then deal with this equation cautiously by further applying various approximations in order to derive an equation of $u(x(t), t)$. Actually, it is unnecessary to follow such a roundabout, cumbersome treatment because what we can know from such a treatment is the velocity corresponding to the fluid momentum, $\frac{p(x(t), t)}{\sqrt{1+p^{2}(x(t), t)}}$, rather than the fluid velocity appearing in Maxwell equations (MEs), i.e, $\frac{j}{n}$.

Eq. (11) and 4 MEs form a closed equation set expressed by Eulerian variables

$\partial_{t} \frac{u}{\sqrt{1-u^{2}}}=-E-u \times B$

$\partial_{t} E=n u+\nabla \times B$

$\nabla \cdot E=-n+Z N_{i}$

$\nabla \times E=-\partial_{t} B$

$\nabla \cdot B=0$.

In addition, we also present a more straightforward process of deriving this equation set in an appendix.

\section{APPLICATIONS}

From Eqs. (12-16), we can make more reliable investigations on both electromagnetic and electrostatic wakefields.

\subsection{3-D Plasma Electrostatic Structure}

Note that Eqs. (12-16) are nonlinear. For example, Eq. (12) is a nonlinear equation of $u$ and Eq. (13) also contains a nonlinear term $n u$. When studying 3-D version of Eqs. (12-16), we should be aware of that due to these nonlinear terms, the solution is unable to have a separable form func $1(r)^{*}$ func $2(\xi)$. Strictly speaking, for any nonlinear differential equation in high-dimensional case, the wellknown method of separation of variables often does not work. Therefore, we treat those related physical quantities appearing in Eqs. (12-16) as having a common form (where $\left.\xi=\eta_{z}-t\right)$

$Q(r, \theta, z, t)=Q(r, z, \xi)$.

Moreover, sometimes the term "electrostatic" is understood loosely as referring to a time-independent $B=\bar{B}(r, z, \theta) \neq 0$. However, such a time-independent $B=\bar{B}(r, z, \theta) \neq 0$, which is "time-dependent" relative to the 
$\frac{1}{\eta}$-frame, does not favor a plasma electrostatic wave whose $E$ is static relative to the $\frac{1}{\eta}$-frame. Unless such a timedependent $B$ is also $z$-independent, otherwise such a running wave form $E=E\left(r, \eta_{z}-t, \theta\right)$ will not appear. This can be verified by strictly analyzing Eqs. (12-16). For a transverse inhomogeneous static $B=\bar{B}(r, \theta) \neq 0$, we can find that there are three corresponding static quantities: $\bar{E}$, $\bar{n}$ and $\bar{u}$ which meet $\bar{E}+\bar{u} \times \bar{B}=0, \nabla \cdot \bar{E}=\bar{n}-\alpha N \quad(\alpha$ is a constant coefficient ) and $\nabla \times \bar{B}=\bar{n} \bar{u}$. An equation of $p$. $\bar{B}$ can be derived in the same way like deriving Eq. (31) (see below). Then, because $\bar{B}$ is $\xi$-independent, we can obtain an equation of $p$ which depends on $\bar{B}$. But we can find that because such a $B=\bar{B}(r, \theta) \neq 0$ does not couple with $\partial_{\xi} p$, it will not affect periodicity requirement $\beta \propto \frac{1}{r}$, which is presented below. A severe constraint on transverse shape for warranting longitudinal periodicity still holds in $B=\bar{B}(r, \theta) \neq 0$ case. Detailed investigations on such a $B=\bar{B}(r, \theta) \neq 0$ case will be presented in other works.

In the 3-D case, we introduce two functions $\beta$ and $\lambda$ to denote the ratio between velocity components along with different directions

$u_{r}=\beta u_{z} ; p_{r}=\beta p_{z}$,

$u_{\theta}=\lambda u_{z} ; p_{\theta}=\lambda p_{z}$.

Eqs. (12-16) will yield following formulas [40]

$E_{z}=-\partial_{t} p_{z}=\partial_{\xi} p_{z} ;$

$E_{r}=\partial_{\xi} p_{r}=\partial_{\xi}\left(\beta p_{z}\right)=\beta \partial_{\xi} p_{z}+\left(\partial_{\xi} \beta\right) p_{z}$,

$E_{\theta}=\partial_{\xi} p_{\theta}=\partial_{\xi}\left(\lambda p_{z}\right)=\lambda \partial_{\xi} p_{z}+\left(\partial_{\xi} \lambda\right) p_{z}$.

$-\partial_{t} B_{\theta}=0=\left[\eta \partial_{\xi} E_{r}-\partial_{r} E_{z}\right]$

$=\eta \partial_{\xi}\left[\beta \partial_{\xi} p_{z}+\left(\partial_{\xi} \beta\right) p_{z}\right]-\partial_{r} E_{z}$

$=\left(2 \eta \partial_{\xi} \beta\right) \partial_{\xi} p_{z}+\eta \beta \partial_{\xi \xi} p_{z}+\eta\left(\partial_{\xi \xi} \beta\right) p_{z}-\partial_{r} \partial_{\xi} p_{z}$.

$-\partial_{t} B_{r}=0=\left[\frac{1}{r} \partial_{\theta} E_{z}-\eta \partial_{\xi} E_{\theta}\right]$

$=\frac{1}{r} \partial_{\theta} E_{z}-\eta \partial_{\xi}\left[\lambda \partial_{\xi} p_{z}+\left(\partial_{\xi} \lambda\right) p_{z}\right]$

$=\frac{1}{r} \partial_{\theta} \partial_{\xi} p_{z}-\left(2 \eta \partial_{\xi} \lambda\right) \partial_{\xi} p_{z}-\eta \lambda \partial_{\xi \xi} p_{z}-\eta\left(\partial_{\xi \xi} \lambda\right) p_{z}$

$$
\begin{aligned}
& -\partial_{t} B_{z}=0=\frac{1}{r}\left[\partial_{r}\left(r E_{\theta}\right)-\partial_{\theta} E_{r}\right] \\
& =\frac{1}{r}\left\{\begin{array}{l}
r \partial_{r}\left[\lambda \partial_{\xi} p_{z}+\left(\partial_{\xi} \lambda\right) p_{z}\right]+\left[\lambda \partial_{\xi} p_{z}+\left(\partial_{\xi} \lambda\right) p_{z}\right] \\
-\partial_{\theta}\left[\beta \partial_{\xi} p_{z}+\left(\partial_{\xi} \beta\right) p_{z}\right]
\end{array}\right\} \\
& =\frac{1}{r}\left\{\begin{array}{l}
{\left[r \lambda \partial_{r} \partial_{\xi} p_{z}-\beta \partial_{\theta} \partial_{\xi} p_{z}\right]+\left[r \partial_{r} \partial_{\xi} \lambda+\partial_{\xi} \lambda-\partial_{\theta} \partial_{\xi} \beta\right] p_{z}} \\
+\left[\begin{array}{l}
r\left(\partial_{r} \lambda\right) \partial_{\xi} p_{z}+r\left(\partial_{\xi} \lambda\right) \partial_{r} p_{z}+\lambda \partial_{\xi} p_{z}-\left(\partial_{\theta} \beta\right) \partial_{\xi} p_{z} \\
-\left(\partial_{\xi} \beta\right) \partial_{\theta} p_{z}
\end{array}\right]
\end{array}\right\}
\end{aligned}
$$

Because of Eqs. $(23,24)$, we can rewrite Eq. (25) as 0

$$
=\left\{\begin{array}{c}
{\left[\begin{array}{c}
r \lambda\left(2 \eta \partial_{\xi} \beta\right) \partial_{\xi} p_{z}+r \lambda \eta \beta \partial_{\xi \xi} p_{z}+r \lambda \eta\left(\partial_{\xi \xi} \beta\right) p_{z} \\
-\beta r\left(2 \eta \partial_{\xi} \lambda\right) \partial_{\xi} p_{z}-\beta r \eta \lambda \partial_{\xi \xi} p_{z}-\beta r \eta\left(\partial_{\xi \xi} \lambda\right) p_{z}
\end{array}\right]} \\
+\left[r\left(\partial_{r} \lambda\right) \partial_{\xi} p_{z}+r\left(\partial_{\xi} \lambda\right) \partial_{r} p_{z}+\lambda \partial_{\xi} p_{z}-\left(\partial_{\theta} \beta\right) \partial_{\xi} p_{z}-\left(\partial_{\xi} \beta\right) \partial_{\theta} p_{z}\right] \\
+\left[r \partial_{r} \partial_{\xi} \lambda+\partial_{\xi} \lambda-\partial_{\theta} \partial_{\xi} \beta\right] p_{z}
\end{array}\right.
$$$$
=\left\{\begin{array}{c}
{\left[\begin{array}{c}
r \lambda\left(2 \eta \partial_{\xi} \beta\right) \partial_{\xi} p_{z}-\beta r\left(2 \eta \partial_{\xi} \lambda\right) \partial_{\xi} p_{z}+r\left(\partial_{r} \lambda\right) \partial_{\xi} p_{z} \\
+r\left(\partial_{\xi} \lambda\right) \partial_{r} p_{z}+\lambda \partial_{\xi} p_{z}-\left(\partial_{\theta} \beta\right) \partial_{\xi} p_{z}-\left(\partial_{\xi} \beta\right) \partial_{\theta} p_{z}
\end{array}\right]} \\
+\left[r \lambda \eta\left(\partial_{\xi \xi} \beta\right)-\beta r \eta\left(\partial_{\xi \xi} \lambda\right)+r \partial_{r} \partial_{\xi} \lambda+\partial_{\xi} \lambda-\partial_{\theta} \partial_{\xi} \beta\right] p_{z}
\end{array}\right\} .
$$

in which all second-order derivative terms of $p_{z}$ disappear. Other MEs can be written as

$$
\begin{aligned}
& {\left[Z N_{i}-n\right]} \\
& =\eta \frac{1}{r} \partial_{\xi}\left[r E_{z}\right]+\frac{1}{r} \partial_{r}\left[r E_{r}\right]+\frac{1}{r} \partial_{\theta} E_{\theta} \\
& =\eta \partial_{\xi \xi} p_{z}+\partial_{r}\left[\beta \partial_{\xi} p_{z}+\left(\partial_{\xi} \beta\right) p_{z}\right]+\frac{1}{r}\left[\beta \partial_{\xi} p_{z}+\left(\partial_{\xi} \beta\right) p_{z}\right] \\
& +\frac{1}{r} \partial_{\theta}\left[\lambda \partial_{\xi} p_{z}+\left(\partial_{\xi} \lambda\right) p_{z}\right] \\
& =\left[\eta \partial_{\xi \xi} p_{z}+\beta \partial_{r} \partial_{\xi} p_{z}+\frac{\lambda}{r} \partial_{\theta} \partial_{\xi} p_{z}\right] \\
& +\left[\left(\partial_{r} \beta+\frac{\beta}{r}+\frac{1}{r} \partial_{\theta} \lambda\right) \partial_{\xi} p_{z}+\left(\partial_{\xi} \beta\right) \partial_{r} p_{z}+\frac{1}{r}\left(\partial_{\xi} \lambda\right) \partial_{\theta} p_{z}\right] \\
& +\left[\partial_{r} \partial_{\xi} \beta+\frac{\partial_{\xi} \beta}{r}+\frac{1}{r} \partial_{\theta} \partial_{\xi} \lambda\right] p_{z} . \\
& {\left.[\nabla \times B]\right|_{z}=0=\partial_{t} E_{z}-n u_{z}=-\partial_{\xi} E_{z}-n u_{z} ;} \\
& {\left.[\nabla \times B]\right|_{r}=0=\partial_{t} E_{r}-n u_{r}=-\partial_{\xi} E_{r}-n u_{r} ;} \\
& {\left.[\nabla \times B]\right|_{\theta}=0=\partial_{t} E_{\theta}-n u_{\theta}=-\partial_{\xi} E_{\theta}-n u_{\theta} ;}
\end{aligned}
$$


where Eqs. (24-26,27,28-30) stand for Farady' law, Gauss' law and Ampere' law, respectively. From Eqs. (20-30), we can obtain

$$
\begin{aligned}
& \partial_{\xi \xi} p_{z}=-n u_{z} \\
& =\left\{\begin{array}{c}
{\left[\eta \partial_{\xi \xi} p_{z}+\beta \partial_{r} \partial_{\xi} p_{z}+\frac{\lambda}{r} \partial_{\theta} \partial_{\xi} p_{z}\right]} \\
+\left[\left(\partial_{r} \beta+\frac{\beta}{r}+\frac{1}{r} \partial_{\theta} \lambda\right) \partial_{\xi} p_{z}+\left(\partial_{\xi} \beta\right) \partial_{r} p_{z}+\frac{1}{r}\left(\partial_{\xi} \lambda\right) \partial_{\theta} p_{z}\right] \\
+\left[\partial_{r} \partial_{\xi} \beta+\frac{\partial_{\xi} \beta}{r}+\frac{1}{r} \partial_{\theta} \partial_{\xi} \lambda\right] p_{z}-Z N_{i}
\end{array}\right\} \\
& \frac{p_{z}}{\sqrt{1+\left(1+\beta^{2}+\lambda^{2}\right) p_{z}^{2}}} \\
& =\left\{\begin{array}{c}
{\left[\left(\partial_{r} \beta+\frac{\beta}{r}+\frac{1}{r} \partial_{\theta} \lambda+2 \eta \beta \partial_{\xi} \beta+2 \eta \lambda \partial_{\xi} \lambda\right) \partial_{\xi} p_{z}\right.} \\
+\left(\partial_{\xi} \beta\right) \partial_{r} p_{z}+\frac{1}{r}\left(\partial_{\xi} \lambda\right) \partial_{\theta} p_{z} \\
{\left[\eta\left(1+\beta^{2}+\lambda^{2}\right) \partial_{\xi \xi} p_{z}\right]} \\
+\left[\partial_{r} \partial_{\xi} \beta+\frac{\partial_{\xi} \beta}{r}+\beta \eta \partial_{\xi \xi} \beta+\frac{1}{r} \partial_{\theta} \partial_{\xi} \lambda+\lambda \eta \partial_{\xi \xi} \lambda\right] p_{z}-Z N_{i}
\end{array}\right\} \\
& \frac{p_{z}}{\sqrt{1+\left(1+\beta^{2}+\lambda^{2}\right) p_{z}^{2}}} \text {. }
\end{aligned}
$$

Likewise, two similar equations for $p_{r}=\beta p_{z}$ and $p_{\theta}=\lambda p_{\theta}$ exist

$-n \beta u_{z}=\partial_{\xi \xi}\left(\beta p_{z}\right)=\beta \partial_{\xi \xi} p_{z}+2 \partial_{\xi} \beta \partial_{\xi} p_{z}+p_{z} \partial_{\xi \xi} \beta$.

$-n \lambda u_{z}=\partial_{\xi \xi}\left(\lambda p_{z}\right)=\lambda \partial_{\xi \xi} p_{z}+2 \partial_{\xi} \lambda \partial_{\xi} p_{z}+p_{z} \partial_{\xi \xi} \lambda$.

and hence there are

$2 \partial_{\xi} \beta \partial_{\xi} p_{z}+p_{z} \partial_{\xi \xi} \beta=0$,

$2 \partial_{\xi} \lambda \partial_{\xi} p_{z}+p_{z} \partial_{\xi \xi} \lambda=0$,

which yields

$\partial_{\xi} \beta=\frac{C_{1}(r, \theta)}{p_{z}^{2}}$ ord $_{\xi} \beta=0$

$\partial_{\xi} \lambda=\frac{C_{2}(r, \theta)}{p_{z}^{2}}$ or $_{\xi} \beta=0$,

where $C_{1,2}(r)$ are binary functions of $r$ and $\theta$.

Obviously, if $p_{z}$ is a periodic function of $\xi$, the equation of $p_{z}$ should correspond to first integral. Because $\beta$ and $\lambda$ appear in Eq. (31), if $\beta$ and $\lambda$ meet the former case $\partial_{\xi} \beta=\frac{C_{1}(r, \theta)}{p_{z}^{2}}$ and $\partial_{\xi} \lambda=\frac{C_{2}(r, \theta)}{p_{z}^{2}}$, Eq. (31) will be very complicated and cannot warrant $p_{z}$ being a periodic function of $\xi$. For finding periodic solutions of Eq. (31), we only need to consider the latter case $\left(\partial_{\xi} \beta, \partial_{\xi} \lambda\right)=(0,0)$ in which $\beta=\beta(r, \theta)$, as well as $\lambda=\lambda(r, \theta)$, are binary functions of $r$ and $\theta$. Thus, we can rewrite Eq. (31) as

$$
\begin{aligned}
& {\left[1-\frac{\eta\left(1+\beta^{2}+\lambda^{2}\right) p_{z}}{\sqrt{1+\left(1+\beta^{2}+\lambda^{2}\right) p_{z}^{2}}}\right] \partial_{\xi \xi} p_{z}} \\
& =\left\{\left[\left(\partial_{r} \beta+\frac{\beta}{r}+\frac{1}{r} \partial_{\theta} \lambda\right) \partial_{\xi} p_{z}\right]-Z N_{i}\right\} \frac{p_{z}}{\sqrt{1+\left(1+\beta^{2}+\lambda^{2}\right) p_{z}^{2}}} .
\end{aligned}
$$

Actually, if our aim is aperiodic electrostatic structure, we can directly start from more general forms of $(\beta, \lambda)$. However, as $p_{z}$ comply with two equations, Eq. (38) and Eq. (26), special attention should be paid to ensure two different equations of the same quantity $p_{z}$ agreeing with each other. Obviously, there are two ways to achieve this goal. The first way is that two equations are alike, i.e., one equation can, by timing a function, become the other. By considering Eqs. (27-30) and verifying different combinations of $\beta$ and $\lambda$, we can find that if $\left(\partial_{\xi} \beta, \partial_{\xi} \lambda\right) \neq(0,0)$, Eqs. (38) and (26) cannot be the same. Therefore, this requires to focus our attention to $\left(\partial_{\xi} \beta, \partial_{\xi} \lambda\right)=(0,0)$ case. The other way is that an equation of $p_{z}$ becomes degenerated (i.e., its coefficients are equal to 0 ). If only Eq. (38) is degenerated then, $p_{z}$ should become a fixed value, and this can be easily verified through Eq. (38). Consequently, when only Eq. (26) is degenerated, two different equations can yield a varying $p_{z}$. By considering Eqs. (27-30) and verifying different combinations of $\beta$ and $\lambda$, we can find that if $\left(\partial_{\xi} \beta, \partial_{\xi} \lambda\right) \neq(0,0)$ exist, Eq. (26) cannot be degenerated. When $\left(\partial_{\xi} \beta, \partial_{\xi} \lambda\right)=(0,0)$, Eq. (26) can be degenerated if $\left[r\left(\partial_{r} \lambda\right)+\lambda-\left(\partial_{\theta} \beta\right)\right]=0$ exists. This also requires us to focus our attention to the $\left(\partial_{\xi} \beta, \partial_{\xi} \lambda\right)=(0,0)$ case. In short, to ensure Eq. (38) and Eq. (26) agree with each other, we should focus our attention to the case of $\left(\partial_{\xi} \beta, \partial_{\xi} \lambda\right)=(0,0)$ and

$\left[r\left(\partial_{r} \lambda\right)+\lambda-\left(\partial_{\theta} \beta\right)\right]=0$.

By re-writing Eq. (38) and timing $\partial_{\xi} p_{z}$ at both sides

$$
\begin{aligned}
& \frac{\partial_{\xi} p_{z} * \partial_{\xi \xi} p_{z}}{\left[\left(\partial_{r} \beta+\frac{\beta}{r}+\frac{1}{r} \partial_{\theta} \lambda\right) \partial_{\xi} p_{z}-Z N_{i}\right]} \\
& =\frac{p_{z} * \partial_{\xi} p_{z}}{\left[\sqrt{1+\left(1+\beta^{2}+\lambda^{2}\right) p_{z}^{2}}-\eta\left(1+\beta^{2}+\lambda^{2}\right) p_{z}\right]},
\end{aligned}
$$


we can find a conservation law

$\xi$ - independentconst

$$
\begin{aligned}
& =\frac{\operatorname{sign}\left(\left(\partial_{r} \beta+\frac{\beta}{r}+\frac{1}{r} \partial_{\theta} \lambda\right) \partial_{\xi} p_{z}-Z N_{i}\right) * Z N_{i}}{\left(\partial_{r} \beta+\frac{\beta}{r}+\frac{1}{r} \partial_{\theta} \lambda\right)^{2}} \\
& \ln \left[\left|\left(\partial_{r} \beta+\frac{\beta}{r}+\frac{1}{r} \partial_{\theta} \lambda\right) \partial_{\xi} p_{z}-Z N_{i}\right|\right]
\end{aligned}
$$

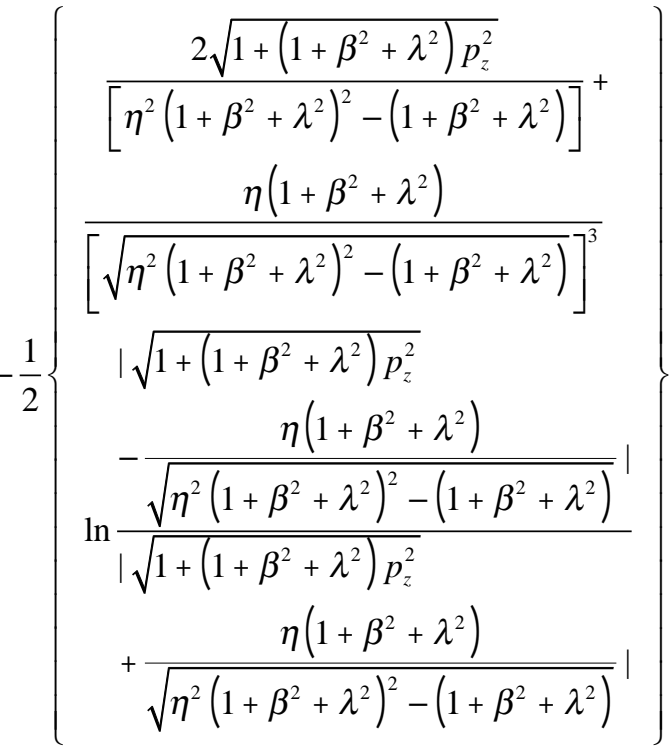

$$
\begin{aligned}
& -\frac{\eta\left(1+\beta^{2}+\lambda^{2}\right)}{\left[\eta^{2}\left(1+\beta^{2}+\lambda^{2}\right)^{2}-\left(1+\beta^{2}+\lambda^{2}\right)\right]} * \\
& {\left[\begin{array}{c}
p_{z}+\frac{1}{2} \frac{1}{\sqrt{\eta^{2}\left(1+\beta^{2}+\lambda^{2}\right)^{2}-\left(1+\beta^{2}+\lambda^{2}\right)}} \\
\ln \frac{1}{\left|p_{z}-\frac{1}{\sqrt{\eta^{2}\left(1+\beta^{2}+\lambda^{2}\right)^{2}-\left(1+\beta^{2}+\lambda^{2}\right)}}\right|} \mid
\end{array}\right]} \\
& +\frac{\partial_{\xi} p_{z}}{\left(\partial_{r} \beta+\frac{\beta}{r}+\frac{1}{r} \partial_{\theta} \lambda\right)} .
\end{aligned}
$$

where $\operatorname{sign}(x)=\frac{x}{|x|}$ is the sign function.

Transverse factors $\beta(r, \theta)$ and $\lambda(r, \theta)$ are determined by the transverse geometrics of the driving stimulus. Moreover, Eq. (40) reveals an important universal property of aperiodic structure. That is, it is possible for a $p_{z}$-value to correspond to different $\partial_{\xi} p_{z}$-values. Because the phase velocity $\frac{1}{\eta}$ is a characteristic constant velocity, there is $\partial_{\xi} p_{z}=0$ for $p_{z}=\frac{1}{\sqrt{\eta^{2}\left(1+\beta^{2}+\lambda^{2}\right)^{2}-\left(1+\beta^{2}+\lambda^{2}\right)}}$ (i.e. $u_{z}=\frac{1}{\eta}$ ). If calculating Eq. (40), we can find, from Fig. (1), that in addition to $\partial_{\xi} p_{z}=0$, there are other three values of $\partial_{\xi} p_{z}$ when $p_{z}=\frac{1}{\sqrt{\eta^{2}\left(1+\beta^{2}+\lambda^{2}\right)^{2}-\left(1+\beta^{2}+\lambda^{2}\right)}}$. The first is greater than $Z N_{i} /\left(\partial_{r} \beta+\frac{\beta}{r}+\frac{1}{r} \partial_{\theta} \lambda\right)$ and denoted as $\left[\left.\partial_{\xi} p_{z}\right|_{u_{z}} \frac{1}{\eta}\right]^{+}$. The second is smaller than $Z N_{i} /\left(\partial_{r} \beta+\frac{\beta}{r}+\frac{1}{r} \partial_{\theta} \lambda\right)$ and denoted as $\left[\left.\partial_{\xi} p_{z}\right|_{u_{z}=} \frac{1}{\eta}\right]^{-}$. The third is smaller than 0 .

Multiple possible values of $\partial_{\xi} p_{z}$ determine very subtle behavior of the aperiodic structure at the neighborhood region of $p_{z}=\frac{1}{\sqrt{\eta^{2}\left(1+\beta^{2}+\lambda^{2}\right)^{2}-\left(1+\beta^{2}+\lambda^{2}\right)}}$ or $u_{z}=\frac{1}{\eta}$. For a periodic structure, the constraint $n \geq 0$ always confines

$$
p_{z}<\frac{1}{\sqrt{\eta^{2}\left(1+\beta^{2}+\lambda^{2}\right)^{2}-\left(1+\beta^{2}+\lambda^{2}\right)}} .
$$

contrast, in an aperiodic structure, multiple possible values of $\partial_{\xi} p_{z}$ at $p_{z}=\frac{1}{\sqrt{\eta^{2}\left(1+\beta^{2}+\lambda^{2}\right)^{2}-\left(1+\beta^{2}+\lambda^{2}\right)}}$ can reach $p_{z} \geq \frac{1}{\sqrt{\eta^{2}\left(1+\beta^{2}+\lambda^{2}\right)^{2}-\left(1+\beta^{2}+\lambda^{2}\right)}} \quad$ regime without violating the constraint $n \geq 0$. For instance, Eq. (40) indicates that if there are

$$
\begin{aligned}
0 & <\sqrt{1+\left(1+\beta^{2}+\lambda^{2}\right) p_{z}^{2}}-\eta\left(1+\beta^{2}+\lambda^{2}\right) p_{z} \rightarrow 0^{+} ; \\
0>\left(\partial_{r} \beta+\frac{\beta}{r}+\frac{1}{r} \partial_{\theta} \lambda\right) \partial_{\xi} p_{z}-Z N_{i} & \rightarrow 0^{-},
\end{aligned}
$$

$\partial_{\xi \xi} p_{z}$ can maintain a finite value and therefore warrant the constraint $n \geq 0$ (because of $\partial_{\xi \xi} p_{z} \neq \infty$ ).

\section{2. phase Space Structure}

According to ref. [40], the phase space profile can be calculated from solved $(E, B)$ :

$$
\begin{aligned}
& f=f_{\text {mопо }}+\sum_{i} b_{i} *\left[(v-u) *\left(v^{2}-1\right)\right]^{i} ; \\
& f_{\text {mono }}=\left\{\int f d^{3} v-\int \sum_{i} b_{i} *\left[(v-u) *\left(v^{2}-1\right)\right]^{i} d^{3} v\right\} * \delta(v-u) .
\end{aligned}
$$



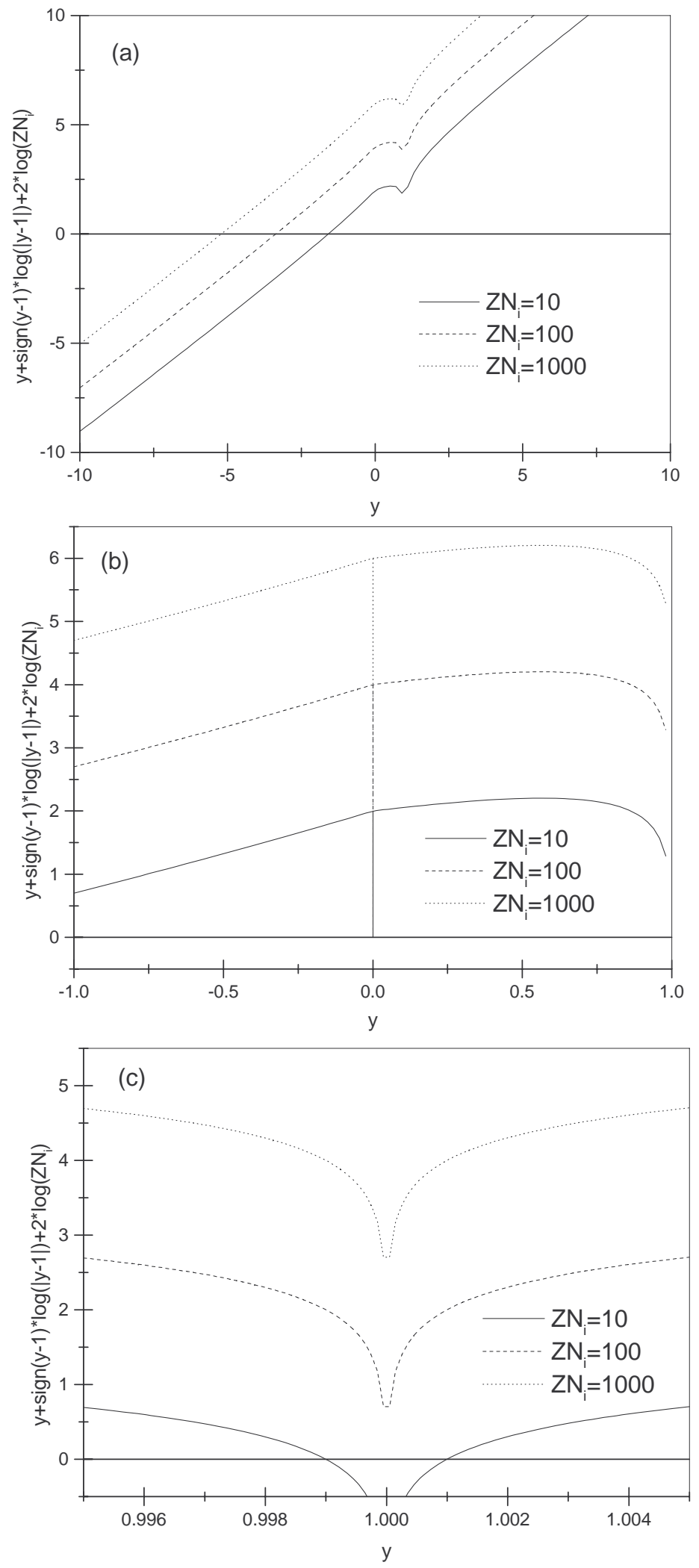

Fig. (1). The behavior of the $\partial_{\xi} p_{z}$-dependent part of Eq. (40) is illustrated by a function $f(y)=y+\operatorname{sign}(y-1) * \log (|y-1|)+2 * \log \left(Z N_{i}\right)$, where $y=\left(\partial_{r} \beta+\frac{\beta}{r}+\frac{1}{r} \partial_{\theta} \lambda\right) \partial_{\xi} p_{z} / Z N_{i}$ and $\operatorname{sign}(x)=\frac{|x|}{x}$. Note that $f(y)$ is the difference between the $\partial_{\xi} p_{z}$-dependent part of Eq. (40) at any $\partial_{\xi} p_{z}$-value and that at $\partial_{\xi} p_{z}=0$ case. (a) is for large-scale global behavior, (b) and (c) are two close-up of the curve in (a) for describing small-scale subtle behaviors nearby $y=0$ and $y=1$ respectively. 
(A)

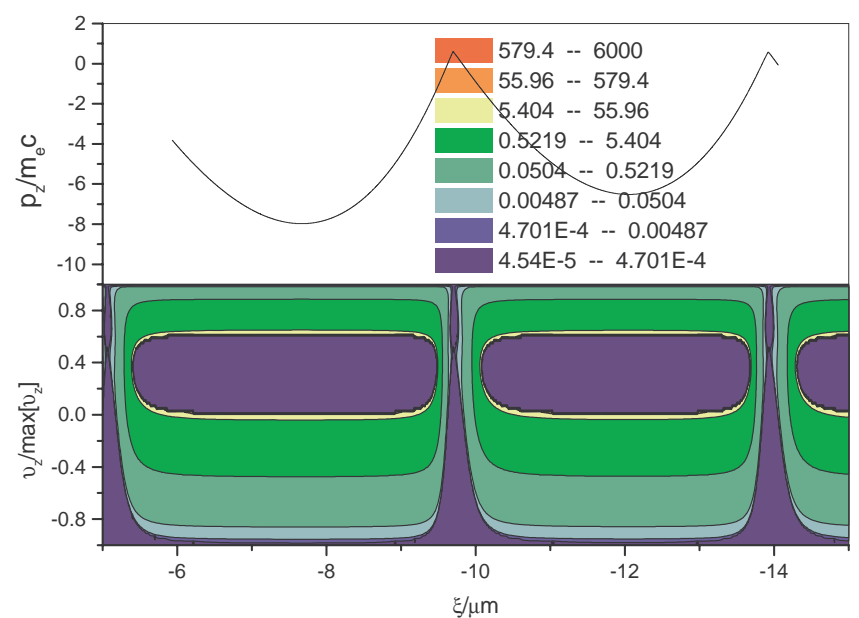

(C)

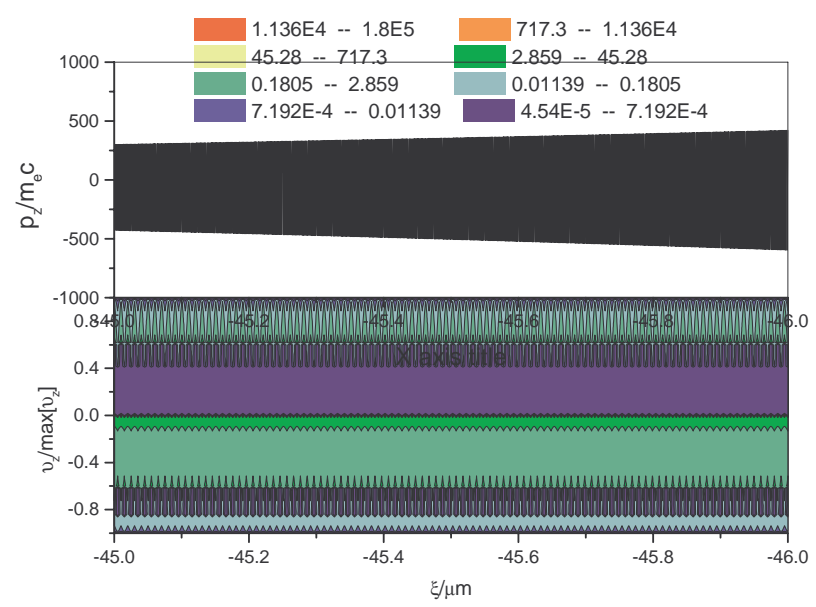

(B)

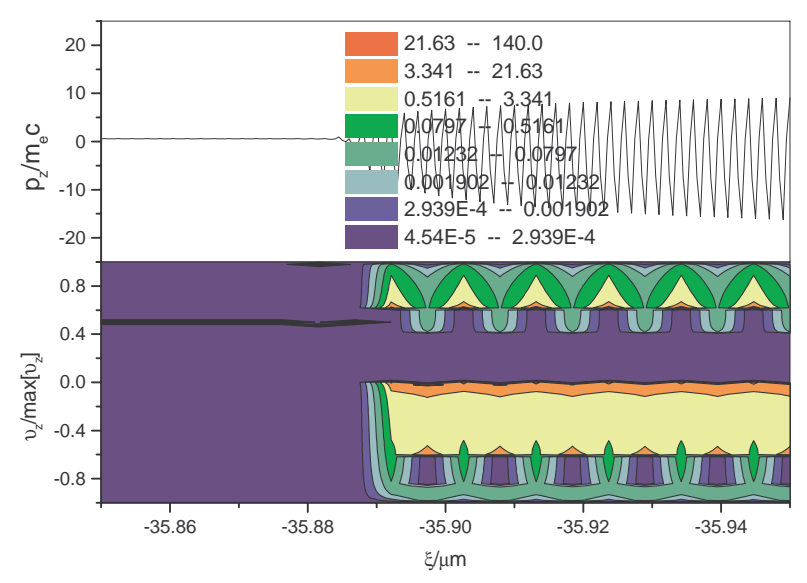

(D)

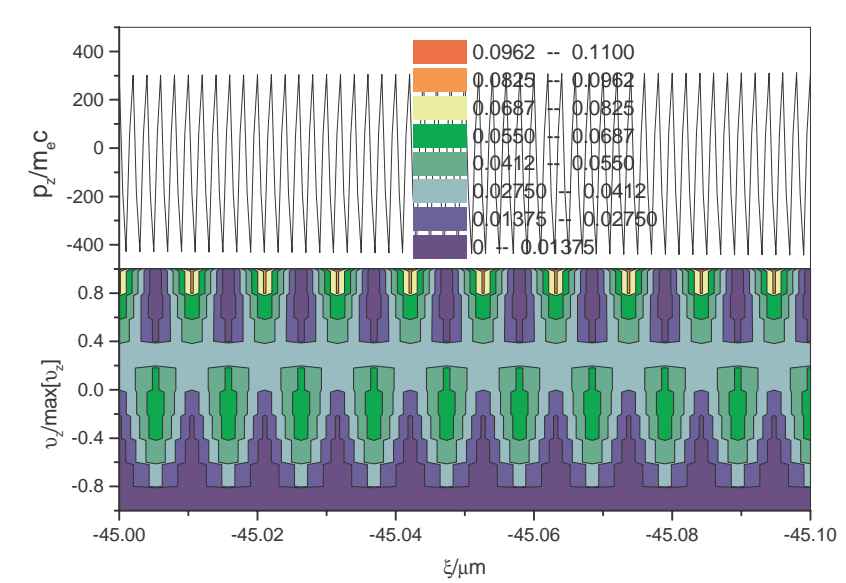

Figs. (2). (A, B, C, D) Examples of contours of $f-f_{\text {mono }}$, where $\max \left[v_{z}\right]=\frac{c}{\sqrt{1+\beta^{2}(r, \theta)}}$ and $E$ has a phase velocity $\frac{1}{\eta}=0.5 c$. The upper panels in Figs. (2-5) reflect the relation between the fluid momentum $p$ and the coordinate $\xi$.

The equation of $b_{i}$ can be obtained by comparing terms in VE

$\partial_{t} b_{i}+u \cdot \nabla b_{i}+\nabla b_{i-1}-b_{i} \nabla u^{\prime}$

$+\frac{1}{\left[\sqrt{1+p^{2}(u)}\right]^{3}} b_{i-1} B \times \frac{[v-u]}{|[v-u]|}=0$.

Strict analysis indicates that the function coefficient set $\left\{b_{i} ; i \geq 1\right\}$ meeting

$b_{2 i-1}=\left[\frac{1}{\eta}-u\right] * c_{i} ;$ and $b_{2 i}=-c_{i}$

where the constant set $\left\{c_{i} ; i \geq 1\right\}$ is independent of spacetime coordinates, is a strict solution of VE in $B=0$ case.

For the aperiodic electrostatic structure, $u$-profile and corresponding space profile in Fig. (2) were plotted. The phase space profiles presented in Fig. (2A-C) also display structures which imply particles acceleration. Here, because $\frac{u_{z}}{\sqrt{1-u \cdot u}}$ display very drastic oscillation at large- $|\xi|$ regime (such as $\xi<-40$ regime in Fig. (2C, D)), for clearance of figures, we plot these profiles over long interval (Fig. 2) and over short interval (Fig. 2B-D), respectively.

Because the aperiodic electrostatic structure is more general than the periodic one, above results seem to suggest a very optimistic prediction of that plasma-based particle acceleration. However, because stimuli driving those wakefields are often magnetized, we should be cautious to treat their wakefields as electrostatic ones. Whether or not these wakefields are electrostatic this should be known through strict initial-value problem calculation of selfconsistent fields, which is given in the next subsection.

\subsection{Electromagnetic Wakefield}

Some authors [12-14] have described wakefields with charge density $n$, which is only related to $E_{\text {long }}$. This 
$n$-description cannot reflect $E_{\text {trans }}$ and $B_{\text {trans }}$ in the region behind the stimulus. Therefore, we started from more basic equation set of $(E, B)$. Moreover, it was noted that when laser-plasma interaction was simulated, the initial condition reflected laser electric field meeting $\nabla \cdot E_{\text {laser }}=0$. If ignoring this constraint and using an initial condition $\nabla \cdot E_{\text {laser }} \neq 0$, we might have unconciously made a "distorted" simulation which cannot correspond to laser-plasma interaction. Which means that, if starting from $\left.\nabla \cdot E\right|_{t=0} \neq 0$, we might have simulated the interaction of a magnetized charged particles beam with plasmas. Such a "distorted" simulation might yield a marked electrostatic wakefield.

Eqs. (12-16) with a constraint $n-Z N_{i}=\partial_{t} \nabla \cdot \frac{u}{\sqrt{1-u^{2}}}+\nabla \cdot[u \times B]=0$ and a condition $N_{i}=0$ describes light (or pure transverse electromagnetic wave) in vacuum. In this situation, the solution of Eqs. (1216) is :

$u=V$ const $=c ; E=-\partial_{t} A ; B=\nabla \times A$,

and $A=\nabla \times \operatorname{Smeets} \nabla \cdot A=0$.

where Vconst represents a constant vector. Likewise, Eqs. (12-16) with a condition $N_{i} \neq 0$ describes light-matter interaction. Strictly speaking, Eqs. (12-16) with a condition $N_{i} \neq 0$ describes the interaction between plasmas and any magnetized charged particles beam. A light beam is a special charged particles beam whose charged density is 0 anywhere and anytime.

Eq. (12) indicates that $E$ can be expressed by $u$ and $B$. This implies that in Eqs. (12-16), only $u$ and $B$ can vary independently. From Eqs. (12-16), we obtain (where $\left.p=\frac{u}{\sqrt{1-u^{2}}}\right)$

$-\partial_{t}\left[u Z N_{i}\right]=\partial_{t t}\left[\partial_{t} p+u \times B\right]-\Delta\left[\partial_{t} p+u \times B\right]$

$+\partial_{t}\left[u \nabla \cdot\left(\partial_{t} p+u \times B\right)\right]+\nabla\left[\nabla \cdot\left(\partial_{t} p+u \times B\right)\right]$

$\operatorname{ord}_{t t}\left[\partial_{t} p\right]-\Delta\left[\partial_{t} p\right]+\partial_{t}\left[u \nabla \cdot\left(\partial_{t} p\right)\right]$

$+\nabla\left[\nabla \cdot\left(\partial_{t} p\right)\right]+\partial_{t}\left[u Z N_{i}\right]$

$=\partial_{t t}[-u \times B]-\Delta[-u \times B]+\partial_{t}[u \nabla \cdot(-u \times B)]$

$+\nabla[\nabla \cdot(-u \times B)]$

If $\theta$ stands for the angle between $u$ and $B$, we can find that in Eq. (48), the left-side terms are $\theta$-independent while the right-side terms will explicitly depend on $\theta$. Thus, for any value of $\sin \theta$, the left-side terms of Eq. (48) remain unchanged. Therefore, it is known that unchanged value of the left-side terms of Eq. (48) by is zero by setting $\theta=0$. It should be noted that $\theta$ has been assumed zero wherever it appears. On the contrary, $\theta$ is solved from the right-side terms of Eq. $(48)=0$. Note that following relation [left - side terms of Eq.(48)]

$=\int[$ left - side terms of Eq.(48) $] \delta(\theta) d \theta$

$=\int[$ right - side terms of Eq. (48) $] \delta(\theta) d \theta=0$,

i.e, $\partial_{t t t} p+\partial_{t}\left[\left(\partial_{t} \nabla \cdot p\right) \frac{p}{\sqrt{1+p^{2}}}\right]+\partial_{t} \nabla \times \nabla \times p+\partial_{t}\left[u Z N_{i}\right]=0$

where $\delta(\theta)$ represents the Dirac function of $\theta$. Subtracting Eq. (47) and Eq. (50), we have

$-\partial_{t}[u \nabla \cdot(u \times B)]-\partial_{t t}[u \times B]-\nabla \times \nabla \times[u \times B]=0$,

which has an obvious solution $u \| B$. Because of Eqs. (1216), this obvious solution implies

$E\|p\| u \| B$ or $E \times B=0$.

$\nabla \times p=\lambda p$, or $p \| \nabla \times p$, where $\lambda$ is a scalar.

i.e., $p \times \nabla \times p=0$.

In addition, another obvious solution $B=0$ just implies unmagnetized charged particles.

Eq. (50) directly implies

$\partial_{t t} p+\left[\left(\partial_{t} \nabla \cdot p\right) \frac{p}{\sqrt{1+p^{2}}}\right]+\nabla \times \nabla \times p=-\left[u Z N_{i}\right]+P O T$

where POT is a constant vector. Moreover, for any charged particles system, there should be a constraint

$n \geq 0 \operatorname{or} \nabla \cdot \partial_{t} p+\nabla \cdot[u \times B]+Z N_{i} \geq 0$.

The initial condition for the light-plasma interaction, if matter is initially a stationary plasma, should read as

$\left.E\right|_{t=0}=E_{\text {light-in-vacuum }}+0$;

$\left.B\right|_{t=0}=B_{\text {light-in-vacuum }}+0 ; 55$

$\left.u\right|_{t=0}=\frac{\text { Vconst } *\left[\nabla \cdot E_{\text {light-in-vacuum }}\right]+0}{\left[\nabla \cdot E_{\text {light-in-vacuum }}\right]+N_{i}}=0$.

Because Eq. (53) is a second-order partial differential equation, there should be another initial condition for $\left.\partial_{t} p\right|_{t=0}$. According to Eq. (12), there is

$\left.\partial_{t} p\right|_{t=0}=\left.E\right|_{t=0}$.

Note that even though $\left.E\right|_{t=0}$ meets $\left.\nabla \cdot E\right|_{t=0}=0$ (because $\left.\left.E\right|_{t=0}=E_{\text {light-in-vacuum }}=-\partial_{t} A=-\partial_{t} \nabla \times S\right), p$ still able to meet $\nabla \cdot p \neq 0$ because $u N_{i}$ meets

$\nabla \cdot u=\frac{1}{\Gamma} \nabla \cdot p+\nabla \frac{1}{\Gamma} \cdot p \neq 0$ evenif $\nabla \cdot p=0$.

and hence is a source responsible for $\nabla \cdot p \neq 0$. This means that, $\nabla \Gamma$ will lead to $\nabla \cdot p \neq 0$ and hence $n-Z N_{i} \neq 0$ (according to Eq. (54)). 
In principle, the light-matter interaction should be treated as an initial-value problem. For any initial condition, we calculate its subsequent evolution described by Eq. (53). Here, for determination of $n$ and $B$ are described as follows:

Eqs. $(47,50)$ indicate that $\partial_{t} p+u \times B$ and $\partial_{t} p$ comply with the same equation. If we apply $\nabla \cdot$ to Eqs. $(47,50)$, we will find that $\nabla \cdot\left[\partial_{t} p+u \times B\right]$ and $\nabla \cdot \partial_{t} p$ also agree with the same equation. This implies a possible relation between $\nabla \cdot\left[\partial_{t} p+u \times B\right]$ and $\nabla \cdot \partial_{t} p$

$\nabla \cdot\left[\partial_{t} p+u \times B\right]=$ function $\left(\left[\nabla \cdot \partial_{t} p\right]\right)$.

One can easily find that if function $(x)=c_{1} x+c_{0}$, where $c_{1}$ and $c_{0}$ are two constants and meet $c_{0}=2 * \max |x|$, $1>c_{1}>-1$,

i.e., $\nabla \cdot \partial_{t} p+\nabla \cdot[u \times B]$

$=2 * \max \left|\left[\nabla \cdot \partial_{t} p+Z N_{i}\right]\right|+\left(1+c_{1}\right) * \nabla \cdot \partial_{t} p$

$=$ cons $\tan t+\left(1+c_{1}\right) * \nabla \cdot \partial_{t} p$

the constraint (54) will always be valid. This just implies

$\partial_{t} p+[u \times B]=$ cons $\tan t *$ Vunit $+\left(1+c_{1}\right) * \partial_{t} p ;$

where $\nabla \cdot$ Vunit $=1$. Here, the constant $c_{1}$ is determined by initial conditions $\left.\partial_{t} p\right|_{t=0},\left.\quad u\right|_{t=0}$ and $\left.B\right|_{t=0}$. Thus, after obtaining $p_{z}$ from Eq. (50), we can solve $B$ according to Eq. (60). Finally, $n$ can also be determined from the solved $p_{z}$ and $B$.

Here, special attention is given to the collective modes of the laser-plasma system. Such a collective mode is characterized by a phase velocity $\frac{1}{\eta}$ and implies $(u, E, B)$ being functions of $\xi=\eta z-t$. People are familar with some typical colloective modes in plasmas, for example, plasma electrostatic wave. Likewise, the collective modes of the laser-plasma system also describe states of the interacting system. For simplicity, here we only consider the case of $p_{\theta}=0, B_{r}=0=B_{z}$, or $p_{r} \neq 0, p_{z} \neq 0$ and $B_{\theta} \neq 0$. From two equations

$\partial_{t t t} p_{r}+\partial_{t}\left[\frac{\left(\partial_{t} \nabla \cdot p\right) p_{r}}{\sqrt{1+p^{2}}}\right]+r-\operatorname{componentof}\left[\partial_{t} \nabla \times \nabla \times p\right]=0 ;$

$\partial_{t t t} p_{z}+\partial_{t}\left[\frac{\left(\partial_{t} \nabla \cdot p\right) p_{z}}{\sqrt{1+p^{2}}}\right]+z-$ componentof $\left[\partial_{t} \nabla \times \nabla \times p\right]=0 ;(62)$

we find that when

$\beta$ is a constant; and $\partial_{r} p_{z}=\beta \partial_{z} p_{z}$ or $p_{z}=p_{z}\left(\beta^{i} \eta^{i} r^{i}+(\eta z-t)^{i}\right)$ exists,

Eqs. $(61,62)$ can be satisfied. More complicated cases in which $\beta$ is a complex space-time function is not considered because it might lead to meaningless solution of $p_{z}$. After straightforward deduction, we re-write Eq. (53) as

$P O T_{z}=\partial_{t t} p_{z}+\left[\left(\partial_{t}\left[\frac{\beta}{r} p_{z}+\beta \partial_{r} p_{z}+\partial_{z} p_{z}\right]\right) \frac{p_{z}}{\sqrt{1+\left(1+\beta^{2}\right) p_{z}^{2}}}\right]$

$+\partial_{z}\left[\frac{\beta}{r} p_{z}+\beta \partial_{r} p_{z}+\partial_{z} p_{z}\right]-\left[\frac{1}{r} \partial_{r} p_{z}+\partial_{r} \partial_{r} p_{z}+\partial_{z} \partial_{z} p_{z}\right]$

$=\left[(\eta \beta)^{-2}-(\eta \beta)^{-1}(1 / \beta+\beta) \frac{p_{z}}{\sqrt{1+\left(1+\beta^{2}\right) p_{z}^{2}}}\right] \beta^{2} \partial_{z z} p_{z}$

$+Z N_{i} \frac{p_{z}}{\sqrt{1+\left(1+\beta^{2}\right) p_{z}^{2}}}$

Here, we focus ourselves on the case of larger phase velocity, i.e. the $0<\eta<1$ case. The so-called conservation law for Eq. (65) reads:

if $\eta^{2}\left(1+\beta^{2}\right)^{2}-\left(1+\beta^{2}\right)<0$, therewillbe

cons $\tan t$

$=\eta^{2} \beta^{2}\left(Z N_{i}-P O T_{z}\right)$

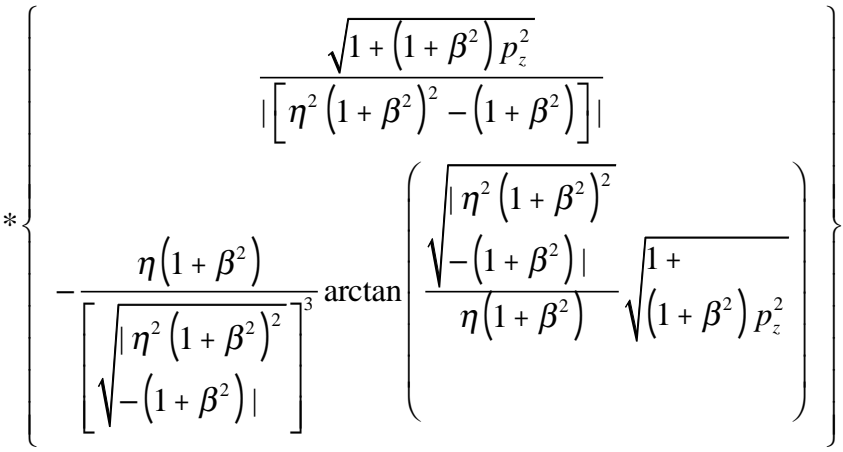

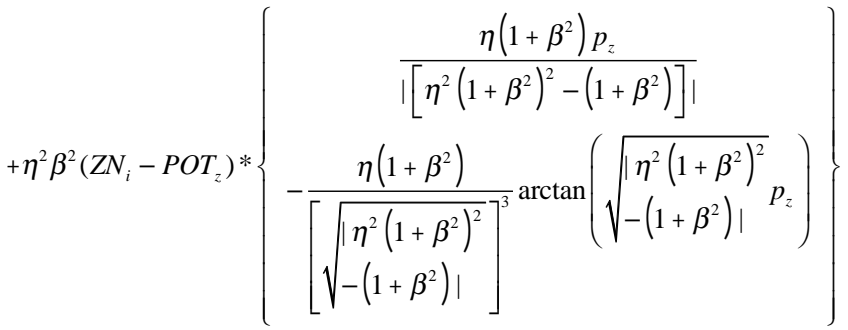

$-\eta^{2} \beta^{2} P O T_{z} * p_{z}+\frac{1}{2}\left[\beta \partial_{z} p_{z}\right]^{2} ;$

if $\eta^{2}\left(1+\beta^{2}\right)^{2}-\left(1+\beta^{2}\right) \geq 0$, there will be constant 


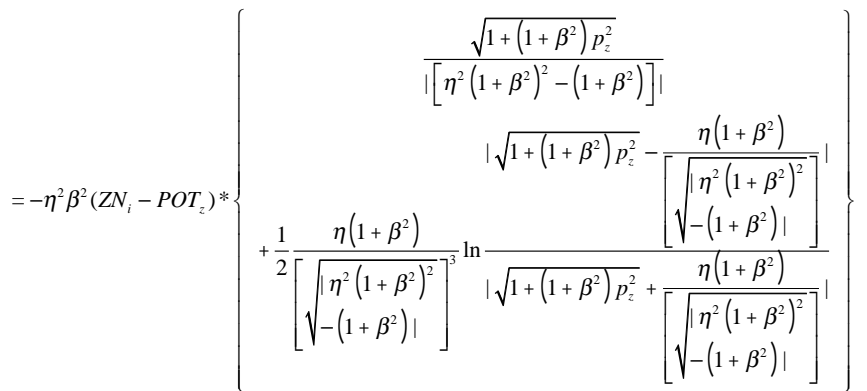

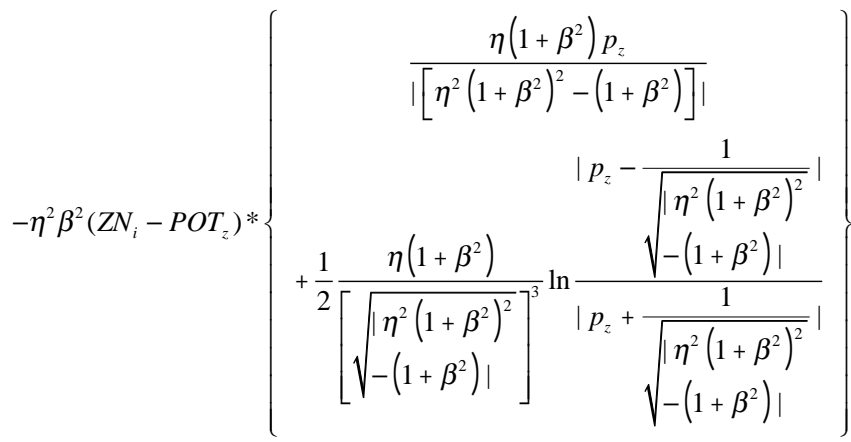

$+\eta^{2} \beta^{2} \operatorname{POT}_{z} * p_{z}+\frac{1}{2}\left[\beta \partial_{z} p_{z}\right]^{2}$.

The value of the constant is determined by the boundary condition at $z=\infty$, which is usually defined as $\left.\partial_{z} p_{z}\right|_{z=\infty}=0$ and $\left.p_{z}\right|_{z=\infty}=0$. From these first integrals in Eqs. $(66,67)$, we can analyze various solutions qualitatively. We calculate these "potential functions" (or $\partial_{z} p_{z}$ independent part) in Eqs. $(66,67)$ and present results in Fig. (3). In terms of mathematics, any solution will correspond to a trajectory on the $\partial_{z} p_{z}-p_{z}$ "phase plane". The abovementioned boundary condition requires that any trajectory must contain the point $\left(\partial_{z} p_{z}, p_{z}\right)=(0,0)$.

We can find that for the case of $P O T=0$ and $\eta^{2}\left(1+\beta^{2}\right)^{2}-\left(1+\beta^{2}\right)<0$, the solution only corresponds to a point in the $\partial_{z} p_{z}-p_{z}$ "phase plane", $\left(\partial_{z} p_{z}, p_{z}\right)=(0,0)$ because at this time, the point $(0,0)$ is the minimum of the "potential function". According to Fig. (3a), $0<P O T<Z N_{i}$ and $\eta^{2}\left(1+\beta^{2}\right)^{2}-\left(1+\beta^{2}\right)<0$ could correspond to a periodic solution (See Fig. 4a). In contrast, for the case $P O T>Z N_{i}$ and $\eta^{2}\left(1+\beta^{2}\right)^{2}-\left(1+\beta^{2}\right)<0$, the solution is aperiodic and corresponds to $n 1260$ over a large-scale region.

The behaviors of these physical quantities depend on the value of $P O T / Z N_{i}$. This agrees with previous discussions. For the periodic solutions, the oscillation of $p_{z}$ agrees with those of $n$ and $B$. For the aperiodic solution, the plots of three physical quantities also have a common trend. That is, after experiencing an extreme value point (maximum or minimum), three quantities monotonically vary with respect to $\xi$. This can only be determined by the potential function curve (the dash dot line) in Fig. (3a).

Above theory and numerical results indicate that if plasma parameter $Z N_{i}$ is given, different POT-values, which can be determined by the initial condition when the EM bunch begins to interact with plasmas, lead to different behaviors of the whole system. From Eqs. (50) and (53), we can find that the $P O T$-value depends on $\left.\partial_{t t} p\right|_{t=0}=\left.\partial_{t t} A\right|_{t=0}=\partial_{t} E_{\text {light-in-vacuum }}$, i.e., the initial shape of the

EM bunch. Because the phase velocity of an EM bunch in vacuum is $c$, there should be $\left.\partial_{t t} p\right|_{t=0}=\left.\partial_{z z} p\right|_{t=0}$. Considering that $P O T$ is a constant, we can define $P O T=\max \left\{\left.\partial_{z z} A\right|_{t=0}\right\}$. As a result, larger value of $\max \left\{\left.\partial_{z z} A\right|_{t=0}\right\}$ will correspond to aperiodic solution in Fig. (4b) whereas smaller value correspond to periodic one in Fig. (4a). This implies that if light pressure is large enough, it is possible to appear as a large scale region in which $n 1260$. This agrees with the so-called "bubble" observed in Particle-in-Cell simulation [37]. Moreover, some authors have found from the PIC simulation, that with the stimulus strength increasing, a transition from periodic wake to "bubble" wake can occur [38]. Above theory confirms such a transition.

On the other hand, another constant parameter $\beta$ should also be determined by initial conditions. Because of its definition $\beta=\frac{p_{r}}{p_{z}}$, we can relate $\beta$ with the ratio between transverse light pressure and longitudinal one, i.e. $\beta=\max \left\{\frac{\left.\partial_{r}|A|^{2}\right|_{t=0}}{\left.\partial_{z}|A|^{2}\right|_{t=0}}\right\}$. Thus, for suitable $\beta$-values, the solution will correspond to Eq. (67). But differing from Eq. (66) where periodic solution corresponds to POT $/ Z N_{i}<1$, Eq. (67) requires periodic solution corresponding to $P O T / Z N_{i}>1$.

From Fig. (4), we can find that both $n$-profile and corresponding $B$-profiles have similar periodic (or aperiodic) structure. These results indicate that the wake of a magnetized stimulus is electromagnetic. Moreover, the phase space profile will assume some hole-like structure and hence suggest particle acceleration. However, for a given $u$ profile, if it is accompanied by a $B$-profile, its accompanying profiles $E$ and $n$, will be affected. As a result, phase space profiles, as well as electron energy spectrum, will be different for respective $B$-profiles. The phase space profiles under different strengths of $B$ are of the same shapes but of different strengths. This implies that the presence of a $B$-profile will affect energy spectrum or acceleration quality. Therefore, when studying plasma-based particle acceleration, one should not automatically take the wakefields as electrostatic one. Otherwise, optimistic overestimation might be obtained. 

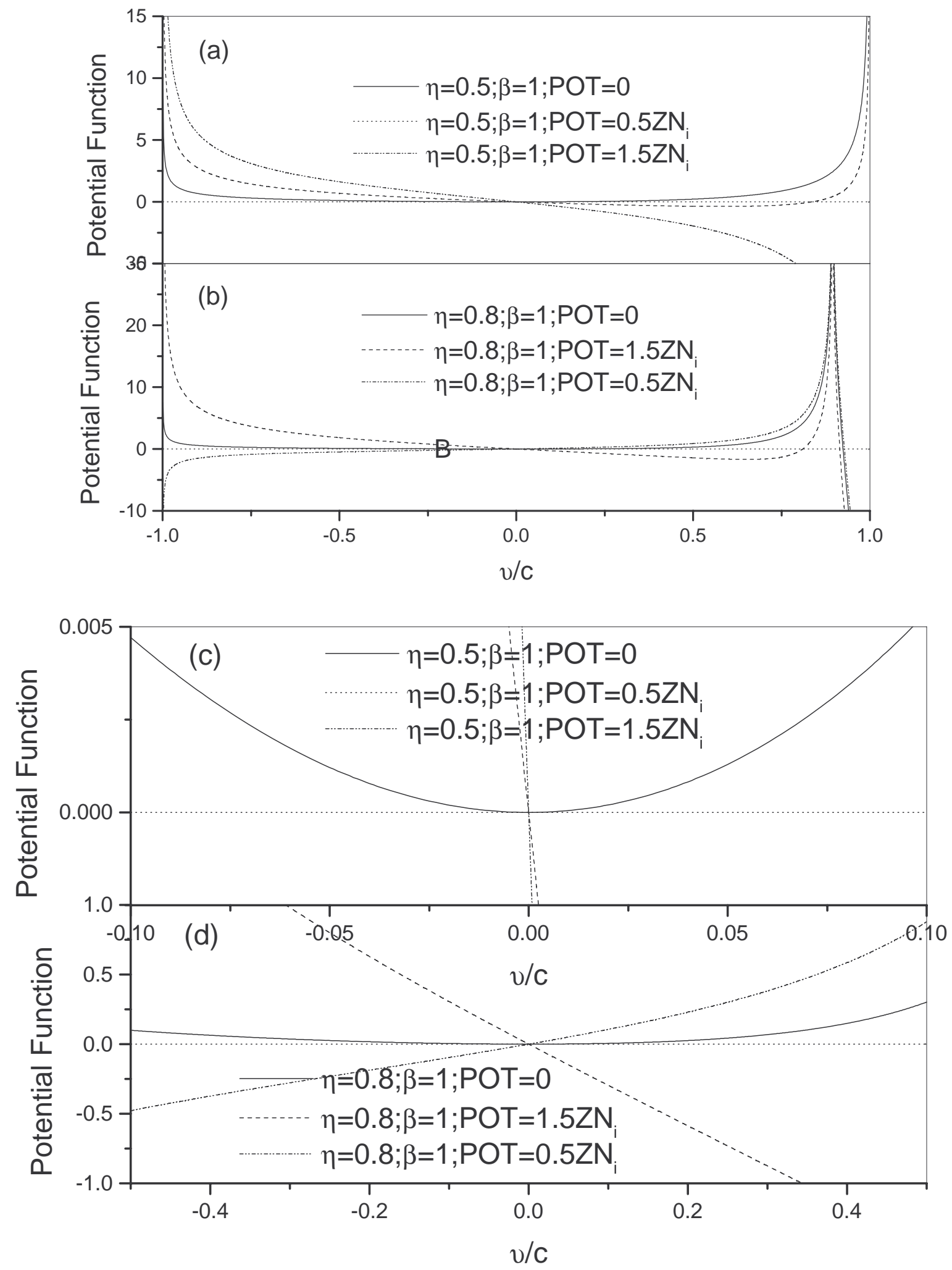

Fig. (3). Examples of the plots of $\partial_{z} p_{z}$-independent part in Eqs. (66-67). (a) is for Eq. (66) and (b) is for Eq. (67). (c) and (d) are local closeups of (a) and (b) respectively. Here, Potential Function is defined as “ $\partial_{z} p_{z}$-independent part in Eq. $\left(6\right.$ ?)” $* \eta^{2} \beta^{2}\left(Z N_{i}-P O T_{z}\right)-“ \partial_{z} p_{z}-$ independent part in Eq. (6) when $p_{z}=0 * * \eta^{2} \beta^{2}\left(Z N_{i}-P O T_{z}\right)$.

Some authors have applied two different methods (plasma cold fluid theory and the PIC algorithm) to study the magnetic field generated from laser-plasma interaction $[41,42]$. Unfortunately, all the initial forms of laser in these 

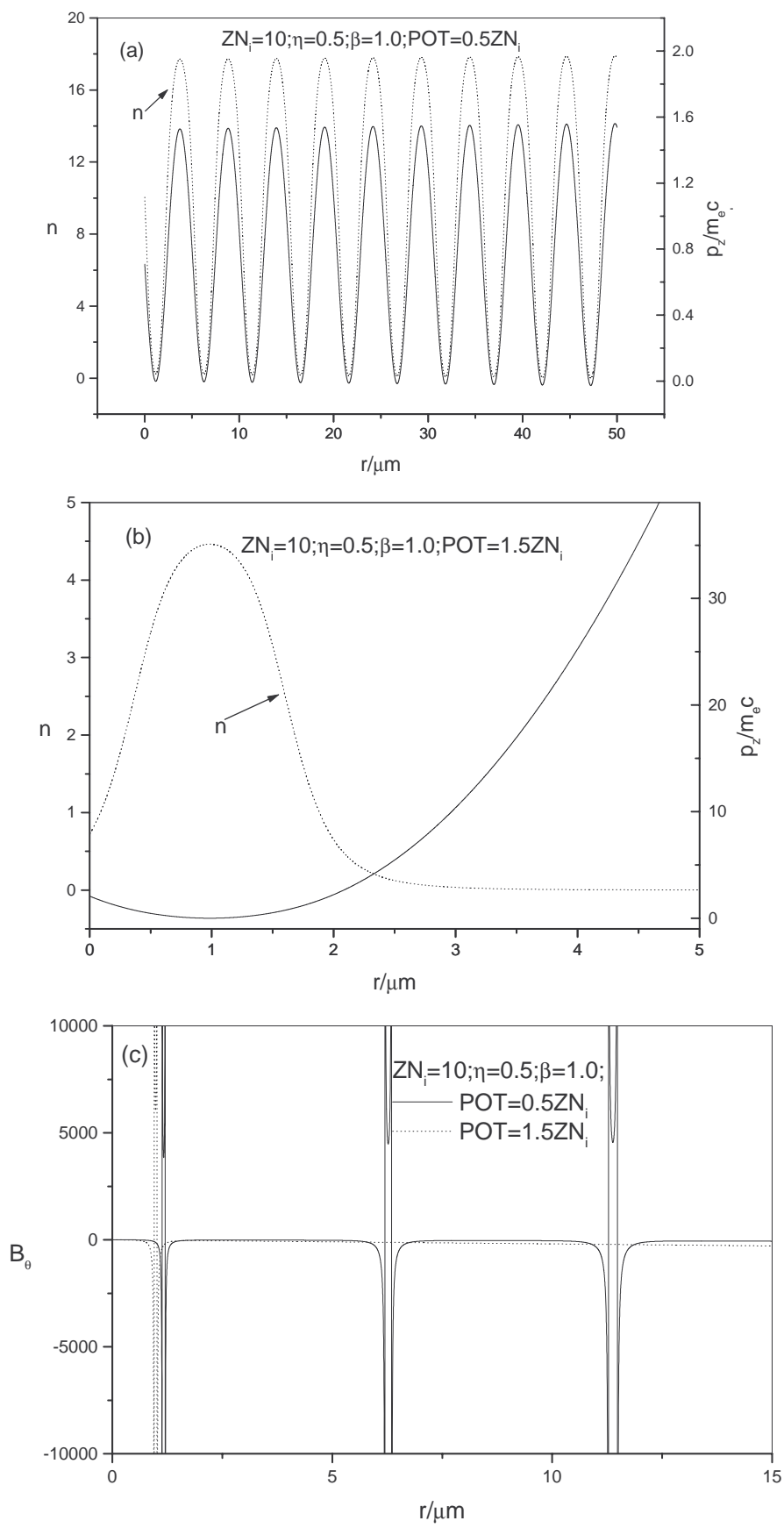

Fig. (4). Examples of $p_{z}$-profiles of plasma electrons and corresponding $n$-profiles and $B_{\theta}$-profiles. Here, $n$ is in unit of $\frac{8.85 * 0.511}{1.6} * 10^{7}[\mu \mathrm{m}]^{-3}, E$ is in unit of $0.511 * 10^{6}[$ Volt $/ \mu \mathrm{m}], B$ is in unit of $0.3 * 0.511 * 10^{6}\left[\right.$ Volt $\left.* f_{s} /(\mu \mathrm{m})^{2}\right]=0.3 * 0.511 * 10^{3}[$ Tesla $]$.

works [41,42] were not seriously considered and met $\nabla \cdot E_{\text {laser }} \neq 0$. Thus, even if the basic theoretical method is perfect, the investigations by these authors will still be away from their purpose because this initial condition makes the investigation not being addressed to the laser-plasma interaction but to the interaction of plasma with a magnetized charged particles beam. These inadequacies leave much room for further improvements in these works.

\section{SUMMARY}

Although the wakes of these stimuli are often expressed by the density waves, such a density wave might be accompanied by a wave of magnetic energy density $B^{2}$. Therefore, the plasma-based accelerator should be viewed as an electromagnetic structure, which is manifested by both the density wave and the magnetic energy density wave. Ignoring the magnetic energy density wave might cause overestimation on plasma-based particle acceleration. 


\section{CONFLICT OF INTEREST}

The authors confirm that they do not have any conflicts of interest.

\section{ACKNOWLEDGEMENTS}

This work is supported by 973 project in China.

\section{APPENDIX}

In the following paragraph, all equations are expressed in terms of Euler variables $(r, t)$, and $v$ is independent of $r$ and $t$.

It is easy to verify once $f$ is a solution of VE with a given pair $(E(r, t), B(r, t))$,

$\left[\partial_{t}+v \cdot \nabla_{r}-[E(r, t)+v \times B(r, t)] \cdot \partial_{p}\right] f(r, v, t)=0$,

where the particle charge $e$ and the particle mass $m$ have been absorbed into $E$ and $B$, and $p=v / \sqrt{1-v \cdot v}$, a zerotemperature type distribution function

$\int f d^{3} v * \delta\left(v-\int v f d^{3} v / \int f d^{3} v\right)=n(r, t) * \delta(v-u(r, t))$

(where $n(r, t)=\int f d^{3} v$ and $\left.u(r, t)=\int v f d^{3} v / \int f d^{3} v\right)$ will meet the following formula

$\left[\partial_{t}+v \cdot \nabla_{r}-[E(r, t)+v \times B(r, t)] \cdot \partial_{p}\right][n * \delta(v-u)]$

$=\left[\partial_{t}+v \cdot \nabla_{r}\right] n * \delta$

$+n *\left\{\left[\partial_{t}+v \cdot \nabla_{r}\right](-u) * \delta^{\prime}-[E(r, t)+v \times B(r, t)] \cdot\left(\partial_{p} v\right) * \delta^{\prime}\right\}$

$=\nabla_{r}[(v-u) n] * \delta+n *\left\{-\int v[E(r, t)+v \times B(r, t)] \cdot \partial_{p} f d^{3} v / \int f d^{3} v\right.$

$+\int v f d^{3} v \int[E(r, t)+v \times B(r, t)] \cdot \partial_{p} f d^{3} v /\left[\int f d^{3} v\right]^{2}$

$\left.-[E(r, t)+v \times B(r, t)] \cdot\left(\partial_{p} v\right)\right\} \delta$

$=\nabla_{r}[(v-u) n] * \delta-n *\left\{\int v[E(r, t)+v \times B(r, t)] \cdot \partial_{p} f d^{3} v / \int f d^{3} v\right.$

$\left.-[E(r, t)+v \times B(r, t)] \cdot\left(\partial_{p} v\right)\right\} \delta^{\prime}$

$=\nabla_{r}[(v-u) n] * \delta-n *\left\{-\int([E(r, t)+v \times B(r, t)]\right.$

$\left.\left[\sqrt{1-v^{2}}\right]^{3}\right) f d^{3} v / \int f d^{3} v$

$\left.+[E(r, t)+v \times B(r, t)] \cdot\left[\sqrt{1-v^{2}}\right]^{3}\right\} \delta^{\prime}$

$=\nabla_{r}[(v-u) n] * \delta+n *\left\{\int([E(r, t)+v \times B(r, t)]\right.$

$\left.\left[\sqrt{1-v^{2}}\right]^{3}\right) f d^{3} v / \int f d^{3} v$

$\left.-[E(r, t)+v \times B(r, t)] \cdot\left[\sqrt{1-v^{2}}\right]^{3}\right\} * d_{v} \delta(v-u)$,

where $\delta$ 'stands for the derivative of the Dirac function $\delta$ with respect to its variable and the relations $x \delta(x)=-\delta(x)$ and $x \delta(x)=0$ have been applied in above formula.
Obviously, Eq. (69) is not a VE of $n(r, t) * \delta(v-u(r, t))$. Actually, it is easier to find above result if we start from Klimontovich-Dupree (K-D) theory [43-45]. According to the K-D theory, a particle system can be described by a function $\quad N(r, v, t)=\sum_{i} \delta\left(r_{t}(t)-r\right) \delta\left(d_{t} r_{i}(t)-v\right) \quad$ which meets the VE. Obviously, a function with more constraint $N_{0}(r, v, t)=\sum \delta\left(r_{t}(t)-r\right) \delta\left(d_{t} r_{i}(t)-v\right) \delta(v-u(r, t))$ will correspond to a zero-temperature distribution function. Even though $\quad N(r, v, t)=\sum_{i} \delta\left(r_{t}(t)-r\right) \delta\left(d_{t} r_{i}(t)-v\right) \quad$ always meet the VE,

$N_{0}(r, v, t)=\sum_{i} \delta\left(r_{t}(t)-r\right) \delta\left(d_{t} r_{i}(t)-v\right) \delta(v-u(r, t))$

does not. Moreover, if trying another form of zerotemperature distribution function $\delta\left(\frac{v-u}{n}\right)$, which can also ensure $\int \delta\left(\frac{v-u}{n}\right) d^{3} v=n$, we can also find that it usually cannot meet the VE.

For any particle system described by a microscopic distribution function $f$, we can always view it as the summation of two subsystems of a same fluid velocity $u$, one consists of all particles whose velocities are equal to $u=\frac{\int v f d^{3} v}{\int f d^{3} v}$ and the other is described by a "hollow" distribution $f_{h o}$ which meets $f_{h o}(r, v=u, t)=0$ and $u=\frac{\int v f_{h o} d^{3} v}{\int f_{h o} d^{3} v}$. Above results revealed that each subsystem does not have a conserved total particle number and hence exchanges particles with the other. This might be the root cause for the zero-temperature type subsystem, which corresponds to $f_{0}=f-f_{h o}$, which does not meet the VE (Eq. (68)). Now that any $f$ can be viewed as $f_{0}+f_{h o}$, it is necessary to find the equation which is satisfied by $f_{0}$. Once this equation is found, an exacter macroscopic equation of $u$ can be found Otherwise, we have to deal with a macroscopic equation derived from the VE.

The microscopic dynamics equation of $f_{0}=n_{0}(r, t) \delta(v-u(r, t))$, where $n_{0}=\int f_{0} d^{3} v$, can be derived straightforward. Clearly, there are

$$
\begin{aligned}
& \partial_{t} f_{0}=\partial_{t} n_{0} * \delta(v-u(r, t))-n_{0} * \partial_{t} u * \delta^{\prime} ; 70 \\
& \nabla_{r} f_{0}=\nabla_{r} n_{0} * \delta(v-u(r, t))-n_{0} * \nabla_{r} u * \delta 71
\end{aligned}
$$

and hence

$$
\begin{aligned}
& {\left[\partial_{t}+v \cdot \nabla_{r}-[E(r, t)+v \times B(r, t)] \cdot \partial_{p}\right] f_{0}} \\
& =\left[\partial_{t} n_{0}+v \cdot \nabla_{r} n_{0}\right] * \delta-n_{0}\left[\partial_{t} u+v \cdot \nabla_{r} u\right] \delta \\
& -[E(r, t)+v \times B(r, t)](\sqrt{1-v \cdot v})^{3} \cdot \partial_{v}\left(n_{0} \delta\right)
\end{aligned}
$$




$$
\begin{aligned}
& =\left[\partial_{t} n_{0}+u \cdot \nabla_{r} n_{0}\right] * \delta-n_{0}\left[\partial_{t} u+v \cdot \nabla_{r} u\right] \delta^{\prime} \\
& -n_{0}[E(r, t)+v \times B(r, t)](\sqrt{1-v \cdot v})^{3} \cdot \delta^{\prime} \\
& =\left[\partial_{t} n_{0}+u \cdot \nabla_{r} n_{0}\right] * \delta-n_{0}\left[\partial_{t} u+v \cdot \nabla_{r} u\right] \delta^{\prime}-n_{0} \\
& {[E(r, t)+u \times B(r, t)](\sqrt{1-u \cdot u})^{3} \cdot \delta^{\prime}}
\end{aligned}
$$

where we have used the relationships $v \cdot \nabla_{r} n_{0} * \delta(v-u(r, t))=u \cdot \nabla_{r} n_{0} * \delta(v-u(r, t)) \quad$ and $\frac{(\sqrt{1-v \cdot v})^{3}-(\sqrt{1-u \cdot u})^{3}}{v-u} * \delta(v-u(r, t))=0 \quad$ etc. We introduce polynom defined as

polynom $=\left[\partial_{t}+v \cdot \nabla_{r}-[E(r, t)+v \times B(r, t)] \cdot \partial_{p}\right]$

$f_{0}+v \cdot \nabla_{r} \frac{\int v f_{0} d^{3} v}{\int f_{0} d^{3} v} \partial_{v} f_{0}$.

It is easy to verify that Eq. (72) can be written as follows and is definitely an equation of polynom

$0=$ polynom $-\left[\int\right.$ polynom $\left.^{*} d^{3} v\right] * \delta+\left[\int\left(\right.\right.$ polynom $\left.\left.^{*} v\right) d^{3} v\right] * \delta$.

Clearly, there is a strict solution of Eq. (74)

$0=$ polynom $=\left[\partial_{t}+v \cdot \nabla_{r}-[E(r, t)+v \times B(r, t)] \cdot \partial_{p}\right] f_{0}$ $+v \cdot \nabla_{r} u \partial_{v} f_{0}$.

Compared with the VE or Eq. (68), there is a new operator $v \cdot \nabla_{r} u \partial_{v}$ appearing in Eq. (75). Due to this new operator, the continuity equation associated with $n_{0}$ becomes

$\partial_{t} n_{0}+u \cdot \nabla_{r} n_{0}=0$

rather than our familiar $\partial_{t} n_{0}+u \cdot \nabla_{r} n_{0}=-n_{0} \nabla_{r} \cdot u$ (i.e. $\left.\partial_{t} n_{0}+\nabla_{r} \cdot\left(n_{0} u\right)=0\right)$. This new operator reflects the subsystem described by $f_{0}$ having particle exchange with other. Specifically, because $E$ is space-time dependent, a charged particle system cannot be at zero-temperature state in which at any space position, all particles have the same velocity. Space-inhomogeneous $E$ will lead to, in some space positions, the temperature derivating from 0 and hence thermal spread in particles' velocities appear (which means some particles being out of the kernel group described by $f_{0}$ and into the hollow group described by $f-f_{0}$ ).

Likewise, following a standard procedure, we can obtain a macroscopic fluid motion equation from Eq. (75)

$\partial_{t} \frac{u}{\sqrt{1-u^{2}}}+E+u \times B=0$.

In contrast, Eq. (68) can yield our familiar fluid motion equation

$$
\begin{aligned}
& \partial_{t} u+\frac{\int(v-u) \nabla_{r}[v f] d^{3} v}{n} \\
& +\frac{\int[E(r, t)+v \times B(r, t)] \cdot\left[\sqrt{1-v^{2}}\right]^{3} * f d^{3} v}{n}=0 .
\end{aligned}
$$

Obviously, two strict equations of $u$ suggest a balance relation

$$
\begin{aligned}
& \frac{\int(v-u) \nabla_{r}[v f] d^{3} v}{n} \\
& =\left[\begin{array}{l}
-\frac{\int[E(r, t)+v \times B(r, t)] \cdot\left[\sqrt{1-v^{2}}\right]^{3} * f d^{3} v}{n} \\
+[E+u \times B]\left(\sqrt{1-u^{2}}\right)^{3}
\end{array}\right],
\end{aligned}
$$

Above strict mathematics theory revealed that the zerotemperature type distribution function $n(r, t) * \delta(v-u(r, t))$ cannot meet the VE. This implies that once we derive a fluid motion equation from the $\mathrm{VE}$, it is inconsistent for us to put this fluid motion equation in the zero-temperature limit. If following this inconsistent treatment, we will find that there will be a convective term remained in the fluid motion equation in the zero-temperature limit.

In short, if $f$ is a strict solution of the VE, we can construct a zero-temperature distribution function $f_{0}$, which corresponds to a fluid velocity which is similar to the one by $f$, according to a standard procedure: $f_{0}=\left[\int f * \delta\left(v-\frac{\int v f d^{3} v}{\int f d^{3} v}\right) d^{3} v\right] \delta\left(v-\frac{\int v f d^{3} v}{\int f d^{3} v}\right)$. From the equation of this microscopic distribution function, we can follow a traditional procedure to derive a new motion equation of $\frac{\int v f d^{3} v}{\int f d^{3} v}$. This new motion equation and 4 MEs can form a closed equation set.

\section{CONFLICT OF INTEREST}

The authors confirm that this article content has no conflict of interest.

\section{ACKNOWLEDGEMENTS}

Declared none.

\section{REFERENCES}

[1] Morse RL, Nielson CW. a) One-, two-, and three-dimensional numerical simulation of two-beam plasmas. Phys Rev Lett 1969; 23: 1087-90. b) Numerical simulation of warm two-beam plasma. Phys Fluids 1969; 12: 2418.

[2] Schamel H. Electron holes ion holes, and double layers. Phys Rept 1986; 140: 161 and references therein.

[3] Korn J, Schamel H. a) Electron holes and their role in the dynamics of current-carrying weakly collisional plasmas. J Plasma Phys 1996; 56: 303-37. b) ibid 1996; 56: 339

[4] Berk HL, Nielsen CE, Roberts KV. Phase space hydrodynamics of equivalent nonlinear systems: experimental and computational observations. Phys Fluids 1970; 13: 980-95. 
[5] Ghizzo A, Izarar B, Bertrand P, et al. Stability of BernsteinGreene-Kruskal plasma equilibria. Numerical experiments over a long time. Phys Fluids 1988; 31: 72.

[6] Dupree TH. a) Theory of phase space density granulation in plasma. Fluids 1972; 15: 334. b) Theory of phase space density holes. ibid 1982; 25: 277; c) Growth of phase space density holes. ibid 1983; 26: 2460 .

[7] Schamel H. a) Stationary solitary, snoidal and sinusoidal ion acoustic waves. Plasma Phys 1972; 14: 905-24. b) Hole equilibria in Vlasov-Poisson systems: a challenge to wave theories of ideal plasmas. Phys Plasmas 2000; 7: 4831.

[8] Schamel J. A modified Korteweg-de Vries equation for ion acoustic wave due to resonant electrons. Plasma Phys 1973; 9: 37787.

[9] Tajima T, Dawson JM. Laser electron accelerator. Phys Rev Lett 1979; 43: 267-70.

[10] Esarey E, Sprangle P, Krall J, Ting A. Overview of plasma-based accelerator concepts. IEEE Trans Plasma Sci 1996; 24: 252-88.

[11] Esarey E, Schroeder CB, Leemans WP. Physics of laser-driven plasma-based electron accelerators. Rev Mod Phys 2009; 81: 122985 .

[12] Rosenzweig JB. a) Nonlinear plasma dynamics in the plasma wakefield accelerator. Phys Rev Lett 1987; 58: 555-58. b) Multiple-fluid models for plasma wake-field phenomena. Phys. Rev A 1989; 40: 5249-55.

[13] Sprangle P, Esarey E, Ting A. a) Nonlinear interaction of intense laser pulses in plasmas. Phys Rev A 1990; 41: 4463-69. b) Nonlinear theory of intense laser-plasma interactions. Phys Rev Lett 1990; 64, 2011-14.

[14] Katsouleas T. Physical mechanisms in the plasma wake-field accelerator. Phys Rev A 1986; 33: 2056-64

[15] Leemans WP, Joshi JC, Mori WB, Clayton CE, Johnston TW. Nonlinear dynamics of driven relativistic electron plasma waves. Phys Rev A 1992; 46: 5112-22.

[16] Krall J, Joyce G, Esarey E. Vlasov simulations of verylargeamplitude-wave generation in the plasma wake-field accelerator. Phys Rev A 1991; 44: 6854-61.

[17] (a) Bulanov SV, Kirsanov VI, Sakharov SA. Excitation of ultrarelativistic plasma waves by pulse of electromagnetic radiation. J Exp Theor Phys Lett 1989; 50: 198; (b) Teychenn T, Bonnaud G, Bobin J. Wave-breaking limit to the wake-field effect in an underdense plasma. Phys. Rev. E 1993; 48: R3248; (c) E. Esarey, A. Ting, P. Sprangle, D. Umstadter, and X. Liu, IEEE Trans. Plasma Sci. 1993; 21: 95; (d) Schroeder CB, Esarey E, and Shadwick BA, Warm wave breaking of nonlinear plasma waves with arbitrary phase velocities. Phys Rev E 2005; 72: 055401(R)

[18] Berezhiani VI, Murusidze IG. (a) Relativistic wake-field generation by an intense laser pulse in a plasma. Phys Lett A 1990; 148: 33840; (b) Interaction of highly relativistic short laser pulses with plasmas and nonlinear wake-field generation. Phys Scr 1992; 45: 87-90

[19] (a) Keinigs R, Jones ME. Two dimensional dynamics of the plasma wakefield accelerator. Phys Fluids 1987; 30: 252-63; (b) Esarey E, Sprangle P, Krall J, Ting A, Joyce G. Optically guided laser wakefield acceleration. Phys Fluids B 1993; 5: 2690-7; (c) Krall J, Ting A, Esarey E, Sprangle P. Enhanced acceleration in a selfmodulated-laser wake-field accelerator. Phys Rev E 1993; 48: 2157-61; (d) Mora P, Antonsen TM. Kinetic modeling of intense, short laser pulses propagating in tenuous plasmas. Phys Plasmas 1997; 4: 217-29.

[20] Lampe M, Joyce G, Slinker SP. Electron hose instability of a relativistic electron beam in an ion focusing channel. Phys Fluids B 1993; 5: 1888-901.

[21] Barov N, Rosenzweig JB. Energy loss of a high-charge bunched electron beam in plasma: analysis. Phys Rev ST Accel Beams 2004; 7: 061301.

[22] Schroeder CB, Esarey E, Shadwick BA, Leemans WP. (a) Trapping, dark current, and wave breaking in nonlinear plasma waves. Phys Plasmas 2006; 13: 033103. (b) Relativistic warm plasma theory of nonlinear laser-driven electron plasma waves. Phys Rev E 2010; 81: 056403.

[23] Chen LJ, Thouless DJ, Tang JM. Bernstein-Greene-Kruskal solitary waves in three-dimensional magnetized plasma. Phys Rev E 2004; 69: 055401(R).
[24] Yazdanpanah J, Anvari A, Samimi J. Warm relativistic fluid description of the laser wake field accelerator. Phys Plasmas 2009; 16: 023104.

[25] (a) Amiranashvili S, Yu MY, Stenflo BLG, Servin M. Nonlinear standing waves in bounded plasmas. Phys Rev E 2002; 66: 046403. (b) Gorbunov LM, Mora P, Ramazashvili RR. Steady ion momentum in nonlinear plasma waves. Phys Rev E 2002; 65: 036401 .

[26] Mangles SPD, Murphy CD, Najmudin Z, et al. Monoenergetic beams of relativistic electrons from intense laser-plasma interactions. Nature (London) 2004; 431: 535-8; (b) Geddes CGR Toth CS, Van Tiborg J, et al. High-quality electron beams from a laser wakefield accelerator using plasma-channel guiding. Ibid 2004; 431: 538; (c) Faure J, Glinec Y, Pukhov A, et al. A laserCplasma accelerator producing monoenergetic electron beams. ibid 2004; 43: 541.

[27] Hogan MJ, Barnes CD, Clayton CE, et al. Multi-GeV energy gain in a plasma-wakefield accelerator. Phys Rev Lett 2005; 95: 054802 .

[28] Lu W, Huang C, Zhou M, Mori WB, Katsouleas T. Nonlinear theory for relativistic plasma wakefields in the blowout regime. Phys Rev Lett 2006; 96: 165002

[29] (a) Lotov KV. Plasma response to ultrarelativistic beam propagation. Phys Plasmas 1996; 3: 2753-9; (b) Lotov KV. Blowout regimes of plasma wakefield acceleration. Phys Rev E 2004; 69: 046405. (c) Lee NC. Envelope equation of electrostatic nonlinear waves in relativistic two-fluid plasmas. Phys Plasmas 2010; 17: 082310; (d) Coffey TP. Large amplitude relativistic plasma waves. Phys Plasmas 2010; 17: 052303; (e)Breaking of large amplitude plasma oscillations. Phys Fluids 1971; 14: 1402.

[30] Trines RMGM, Norreys PA. Wave-breaking limits for relativistic electrostatic waves in a one-dimensional warm plasma. Phys Plasmas 2006; 13: 123102 .

[31] (a) Tzoufras M, Lu W, Tsung FS, et al. Beam loading by electrons in nonlinear plasma wakes. Phys Plasmas 2009; 16: 056705. (b) Johnston TW, Tyshetskiy Y, Ghizzo A, Bertrand P. Persistent subplasma-frequency kinetic electrostatic electron nonlinear waves. Phys Plasmas 2009; 16: 042105

[32] Buck A, Nicolai M, Schmid K, et al. Real-time observation of laser-driven electron acceleration. Nat Phys 2011; 7: 543-8

[33] Kaluza MC, Thomas AGR, Mangles SPD, et al. Measurement of magnetic-field structures in a laser-wakefield accelerator. Phys Rev Lett 2010; 105: 115002.

[34] (a) Drake JF, Swisdak M, Cattell C, Shay MA, Rogers RN, Zeiler A. Formation of electron holes and particle energization during magnetic reconnection. Science 2003; 299: 873-7; (b) Drake JF, Shay MA, Thongthai W, Swisdak M. Production of energetic electrons during magnetic reconnection. Phys Rev Lett 2005; 94: 095001; (c) Drake JF, Swisdak M, Chen H, Shay MA. Electron acceleration from contracting magnetic islands during reconnection. Nature 2006; 443: 553-6.

[35] Chen LJ, Bhattacharjee A, Puhl-Quinn PA, et al. Observation of energetic electrons within magnetic islands. Real-time observation of laser-driven electron acceleration. Nat Phys 2008; 4: 19-23.

[36] Hoshino M, Mukai T, Terasawa T, et al. Suprathermal electron acceleration in magnetic reconnection. J Geophys Res 2001 106(25): 979.

[37] Pukhov A, Meyer-ter-Vehn J. Relativistic magnetic self-channeling of light in near-critical plasma: three-dimensional particle-in-cell simulation. Phys Rev Lett 1996; 76: 3975-8.

[38] Geissler M, Schreiber J, Meyer-ter-Vehn J. Bubble acceleration of electrons with few-cycle laser pulses. New J Phys 2006; 8: 186.

[39] Bulanov SV, Lontano M, Esirkepov TZ, et al. Electron vortices produced by ultraintense laser pulses. Phys Rev Lett 1996; 76: 3562

[40] Lin H. Phase space coherent structure of charged particles system. Phys Plasmas 2011; 18: 062107.

[41] Gorbunov LP, Mora P, Antonsen TM. (a) Magnetic Field of a Plasma Wake driven by a Laser Pulse. Phys Rev Lett 1996; 76 : 2495; (b) Quasistatic magnetic field generated by a short laser pulse in an underdense plasma. Phys Plasmas 1996; 4: 4358.

[42] Sheng ZM, Meyer-ter-Vehn J, Pukhov A. Analytic and numerical study of magnetic fields in the plasma wake of an intense laser pulse. Phys Plasmas 1997; 5, 3764. 
[43] (a) Lamb SH. Chap. 1, Hydrodynamics. UK: Cambridge University Press 1932; (b) McCormack PC, Lawrence Crane. Chap. 3, Physical Fluid Dynamics. USA: Academic Press 1973; (c) Landau LD, Lifshitz EM. Chap. 1, Fluid Mechanics. UK: Pergamon 1975.
[44] Davidson RC. Methods in nonlinear plasma theory, Chap 3. USA: Academic Press, 1972; (b) Davidson RC, Schram PP. Nucl Fusion 1968; 8: 183

[45] Krall NA, Trivelpiece AW. Principles of plasma physics. McGrawHill: New York 1973.

Received: July 22, 2013

(C) Lin et al.; Licensee Bentham Open.

This is an open access article licensed under the terms of the Creative Commons Attribution Non-Commercial License (http://creativecommons.org/licenses/by-nc/3.0/) which permits unrestricted, non-commercial use, distribution and reproduction in any medium, provided the work is properly cited. 